\title{
Comparison of unit cell-based computational methods for predicting the strength of wood
}

\author{
J. Füssl ${ }^{\mathrm{a}, 1}$, M. Li ${ }^{\mathrm{a}}$, M. Lukacevic ${ }^{\mathrm{a}}$, J. Eberhardsteiner ${ }^{\mathrm{a}}$, C.M. Martin ${ }^{\mathrm{b}}$ \\ ${ }^{a}$ Vienna University of Technology, Karlsplatz 13, 1040 Vienna, Austria \\ ${ }^{b}$ Department of Engineering Science, University of Oxford, Parks Road, Oxford, OX1 3PJ, U.K.
}

\begin{abstract}
Wood, as a naturally-grown material, exhibits a highly anisotropic and inhomogeneous material structure, with a complex wood fibre distribution influenced by randomly occurring knots. Thus, for the prediction of effective strength properties of wood, advanced computational tools are required, which are able to predict as well as consider multidimensional strength information at different scales of observation.

Within this work, three such computational methods will be presented: an extended finite element approach able to describe strong strain-softening and, thus, reproduce brittle failure modes accurately; a newly-developed limit analysis approach, exclusively describing ductile failure; and an elastic limit approach based on continuum micromechanics. All three methods are applied to earlywood and latewood unit cells and to clear wood, finally yielding effective failure surfaces for a range of multidimensional stress states. These failure surfaces are compared with each other and with experimental results from biaxial tests. Based on these comparisons, the strengths and weaknesses of the three computational methods are discussed, and their applicability to wood is evaluated.

The extended finite element method is a powerful technique that allows for a very realistic description of strength-governing processes. Nevertheless, its complexity and high computational effort prevent widespread use in the engineering field. The plastic limit analysis and elastic limit approaches, however, show good predictive performance compared with the extended finite element method, coupled with excellent efficiency and stability. In this study it is found that together, the latter two approaches are able to enclose the experimentally-obtained failure regions for clear wood almost perfectly, while also delivering new insights with respect to the ductile failure potential of wood.

The conclusion can be drawn that there exist promising computational methods that are capable of delivering reliable multidimensional strength information for wood and, subsequently, will enable effective strength predictions for wooden boards and wood-based products. Finally, this work is intended as a contribution to performance-based optimisation of wooden structures, a necessity for wood to become competitive with respect to other building materials.
\end{abstract}

Keywords: Wood, Strength, XFEM, Limit Analysis, Elastic Limit Estimates

2010 MSC: 00-01, 99-00

\footnotetext{
* Corresponding author

Email address: josef.fuessl@tuwien.ac.at (J. Füssl)

$U R L$ : www.imws.tuwien.ac.at (J. Füssl)
} 


\section{Introduction}

Traditionally, wood as a structural building material has mainly been used in rural areas for one- or two-storey residential buildings or simple halls and stables. Due to the relatively small dimensions of such buildings and the fact that each structural element only appears in a small number, it has not been necessary to exploit the full mechanical potential of wood. Simple design rules combined with practical experience and considerably oversized components have together met all requirements.

In recent years, this situation has changed dramatically. The excellent mechanical and physical properties of wood, combined with the general trend of growing environmental awareness, have put timber structures into the focus of private as well as public building developers - not just to realise small buildings, but to use wooden building elements for highly sophisticated engineering structures. There has already been a nine-storey tower built in London and a 12-storey wooden building is under construction in Bergen, Norway. A 24-storey wooden skyscraper will be completed in Vienna in 2018, which, with a height of $84 \mathrm{~m}$, will be the tallest wooden skyscraper in the world. Another wooden tower, comprising 35 storeys, is planned in Paris, aimed at addressing the French capital's housing challenges in a sustainable, creative and environmentallyfriendly manner. Meanwhile, a 34-storey wooden apartment block could be built in Stockholm by 2023 if the planning authorities have their way.

Such projects could, or respectively can, only be realised by the strong initiative of individual responsible authorities, and all of these developers struggle to justify their wooden buildings on economic grounds. Indeed, it is only possible for timber structures to be cost-competitive with steel or concrete structures under ideal planning and executing conditions. A major reason for this is the aforementioned traditional origin of timber constructions, the associated simple design practice, and the resulting conservative dimensioning of wooden structural elements.

This has been the motivation for the present work, which aims at the development and assessment of new computational methods enabling better predictions of the mechanical behaviour of wood. Based on such methods, the great mechanical potential of the 'raw' wooden material should be much better utilised. In this context, simple wooden boards, obtained in sawmills by cutting of logs, are considered as 'raw' material. For use in construction, these wooden boards are typically assembled into wood-based products like glued-laminated timber (GLT) or cross-laminated timber (CLT). The basic steps of this procedure are shown in Figure 1, together with the principal material directions of the flawless wood. The tubular structure of the wooden cells proceeds in the $L$-direction (longitudinal fibre direction), the $R$-direction denotes the radial direction with respect to the central pith, and the $T$-direction describes the tangent direction to the circular annual rings. These directions of the flawless wood, which is subsequently referred to as clear wood, are disturbed by randomly occurring knots, forcing the fibres to deviate from the global longitudinal direction. Such knots or knot groups, as illustrated in Figure 1, introduce large fluctuations of wooden board properties, and in general board sections with knots exhibit poorer mechanical behaviour than clear wood sections. For this reason, knot-related characteristics are commonly used for sorting and classifying wooden boards. The more accurately this classification procedure works, the more efficiently wooden boards can be used in wood-based products.

Unfortunately, only empirically derived relationships between knot characteristics and certain board 

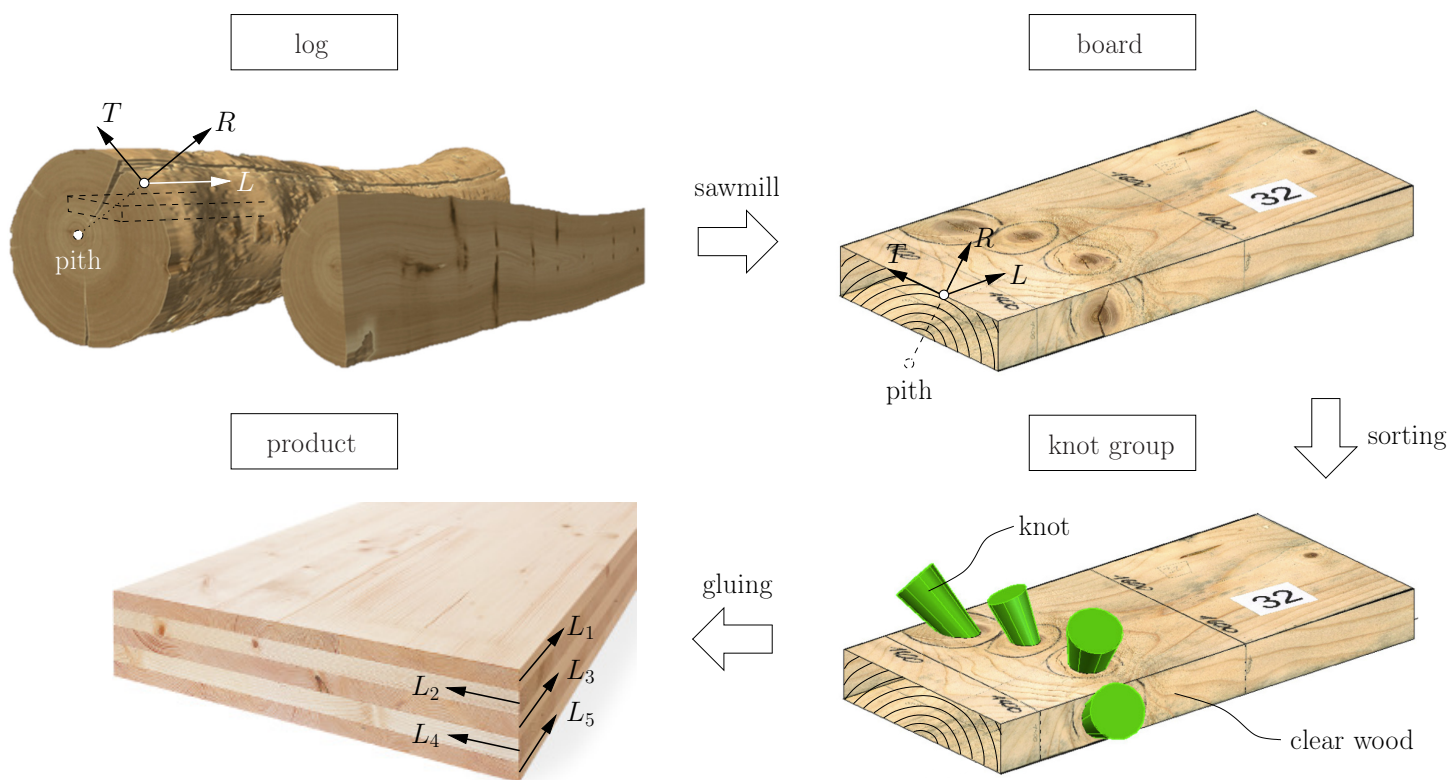

Figure 1: Basic steps in wood processing. Wooden boards are obtained in sawmills by cutting logs. These boards are sorted and rated according to characteristics like knots and fibre deviations, before they are glued together to create products (like the CLT board shown). as the fibre distribution around them, as shown schematically in Figure 2. Suddenly, timber design, which

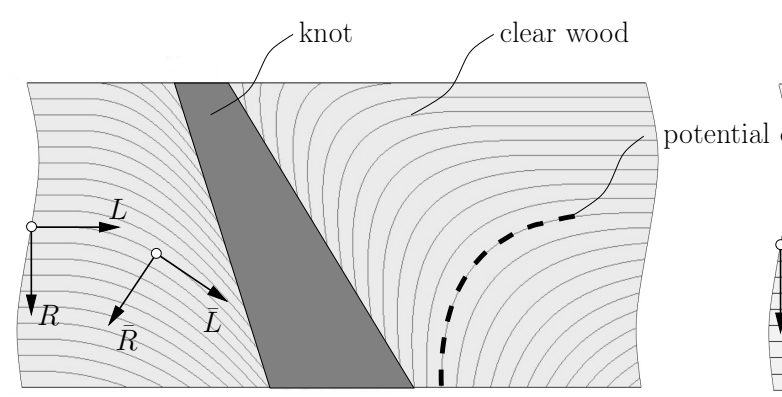

(a)

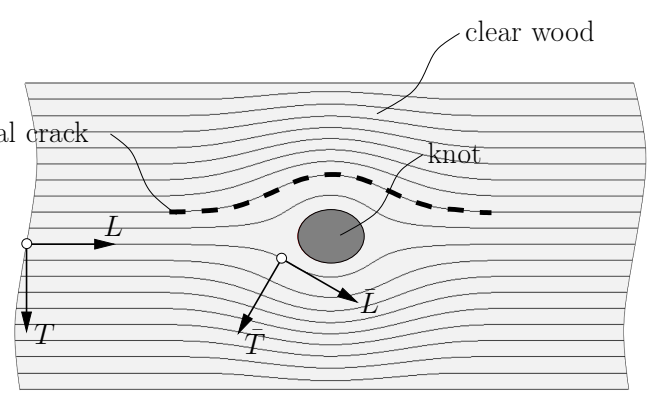

(b)

Figure 2: Fibre distribution around a knot, illustrated in the (a) longitudinal-radial $(L-R)$ plane and (b) longitudinal-tangential $(L-T)$ plane, introducing a variety of different 3D stress states into the clear wood.

properties have been developed and used in the field so far. These do not show very good prediction quality, especially when it comes to strength properties. This is why a growing amount of effort is being put into the development of numerical simulation methods able to describe the influence of knots and knot groups on the effective behaviour of the associated wooden board section. These simulations need to model knots as well

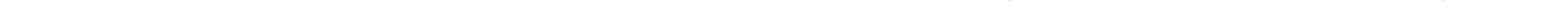

$$
\text { into the clear wood. }
$$

has historically been concerned mostly with beam-like structures, and thus 1D design concepts, has to deal with complex 3D stress and strain fields arising in the vicinity of knots. These stress distributions around knots are often responsible for the initiation of cracks or plastified material zones, and therefore have to be 
taken into account accurately. This is only possible when a detailed characterisation of the multidimensional strength behaviour of the considered clear wood is available. This is actually never the case, because the enormous experimental program that would be required is hardly feasible. Furthermore, it would be virtually impossible to determine experimentally the influence of density fluctuations, different moisture contents, and the different characteristics of several wooden species on the multidimensional strength behaviour.

For this reason, the present work focuses on a new approach for determining 2D and 3D strength information for clear wood. Since failure and plastification is strongly influenced by the complex material system of wood, exhibiting cellular and layered structures on different length scales, a conceptual framework in which these different microstructural characteristics are incorporated appears to be necessary. Since the strength behaviour of the individual components of the wood material can be assumed to be far less complex than that of the overall material system, linking microstructural characteristics to each other and to the macroscopic behaviour, respectively, ultimately leads to a significant reduction of the independent unknown material properties. Moreover, the influence of microstructural changes on the macroscopic behaviour can be identified easily, without performing additional experiments.

Thus, the division of wood into meaningful levels of observation is the first objective of the present work. At each level, failure modes and failure stress states (strength properties) are determined using various computational methods, and the obtained information is transferred - and serves as input - to the next higher level of observation. For this so-called upscaling, a numerical approach based on the extended finite element method has been presented in previous papers of two of the authors [1,54], which is able to describe failure (even cracking mechanisms) of wood very accurately. For a comprehensive description of the strength behaviour over several levels of observation, however, this method alone seems likely to be insufficient and inefficient. Therefore, within the present work, a plastic limit analysis approach is developed and applied to wood at two levels of observation, contributing successfully to the overall multiscale concept for the prediction of strength properties and failure mechanisms. Moreover, both of these numerical methods are compared to an analytical approach based on continuum micromechanics, originally presented by one of the authors in [3], delivering elastic limit stresses for given loading states. These stresses refer to ultimate strength in the case of brittle failure, and to elastic limit stresses in the case of ductile failure. Since the extended finite element method is used to describe brittle failure accurately, and limit analysis is ideally suited for predicting ultimate strength in the case of ductile failure modes, a comprehensive set of complementary methods is available to address the described challenge.

In summary, the objectives of the present work can be formulated as follows:

- Subdividing clear wood into appropriate observation scales and specifying representative unit cells to which the extended finite element method (XFEM), limit analysis (LA) and elastic limit (EL) approaches can be applied.

- Deriving an appropriate LA formulation which is applicable to a unit cell-type homogenisation procedure, similar to the concepts already proposed in $[4,5,6]$, and applying all three methods to the same clear wood material.

- Comparing the effective strength predictions obtained at the different levels of observation, and evaluating the three methods with respect to their capabilities and efficiency. 
- Performance assessment of the three methods with respect to their application to wood, by means of comparisons with experimental results at the clear wood scale.

The paper is structured as follows. In Section 2, the three methods of choice are briefly presented, and the necessary extensions of conventional LA formulations, to be applicable to unit cells of wood microstructures, are given in more detail. Furthermore, an attempt is made to discuss and clarify the fundamental differences between the three methods. Section 3 describes the considered wood species, its microstructure, and the observation scales and representative unit cells derived therefrom. The model geometries and input parameters for all methods are given, and the calculation program is specified. In Section 4, the results of all methods are presented, compared with each other and discussed. Moreover, the results from all three methods at the clear wood scale are plotted against experimental results obtained from biaxial testing. Finally, concluding remarks and a short statement on future work are given in Section 5 .

\section{Methods and theory}

The failure behaviour of wood is quite complex, being strongly dependent on the type of loading and the level of consideration (observation scale). The majority of the models in the scientific literature focus on the wooden board scale (as illustrated in Figure 2), describing the failure behaviour of clear wood and the influence of knots. At this scale, under pressure-dominated stress states, wood behaves in a very ductile manner and strain hardening may even occur. Under tensile- and shear-dominated stress states, a rather brittle failure behaviour can be observed, but with a fracture toughness which can be very different dependent on the wooden structure considered. A brief overview of some existing methods for predicting/modelling structural behaviour at the wooden board level is given in the following.

The first group of approaches avoids any direct description of failure mechanisms, and instead uses mean stress concepts [7,8], where averaged stresses over a small finite volume are assumed to indicate failure. These volumes can be adjusted to typical features of wood, such as structural characteristics of wood fibres [9]. In [10, 11, 12] this approach was applied in combination with findings of linear elastic fracture mechanics. The suitability of different volume definitions, over which the stresses are averaged, and also the effectiveness of various failure criteria, was compared in [13]. A physically-based structural failure criterion was presented in $[14,15]$, where it was assumed that in wooden boards with knots, global failure can be related to a stress transfer mechanism, which is identifiable by evaluating averaged stress fields in the vicinity of knots. Models based on mean stress concepts are mostly dependent on empirical parameters, and the true failure mechanisms cannot be mapped. This can be overcome by directly modelling failure processes.

The most sophisticated approaches for this are based on multi-surface yield/failure criteria, as described in [16]. In these approaches, orthotropic yield surfaces are defined to describe the onset of ductile failure, whereas orthotropic crack surfaces indicate stress states under which cracks are to be expected. Cracking is normally modelled with cohesive elements, including an anisotropic traction separation law for wood. Applications of this model show promising results regarding the estimation of load-carrying behaviour [17, 18]. A cohesive zone model based on plasticity theory has also been used for parametric studies of a gluedlaminated timber beam with a hole [19]. 
These approaches work well for a uniform fibre orientation and if the location of the crack path is known in advance. However, due to knots and the fibre deviations around them, as described in Section 1, complex stress states usually prevail, and such an approach becomes impossible to apply. Thus, to be able to assign reliable failure surfaces and corresponding crack directions to clear wood at the wooden board scale, a multiscale approach is pursued. For such an approach, failure mechanisms at different observation scales need to be analysed. This is done here by using three different promising computational methods. The basic characteristics of each method are described in the following, and are qualitatively shown in Figure 3 . The type of structural response that can be described by each method, in terms of relationships between the effective stress tensor $\boldsymbol{\Sigma}$ and the effective strain tensor $\mathbf{E}$, is illustrated in Figure 3(a), where $\mathbf{C}^{\text {eff }}$ denotes the effective elastic stiffness tensor. Figure 3(b) shows the corresponding relationships between microscopic stresses $\boldsymbol{\sigma}\left(\mathbf{x}_{i}\right)$ and microscopic strains $\varepsilon\left(\mathbf{x}_{i}\right)$, for a material point $i$ of the considered structure. The values given therein denote strength and stiffness values of the wood microstructure (see Figure 6), which will be explained in detail in Section 3.

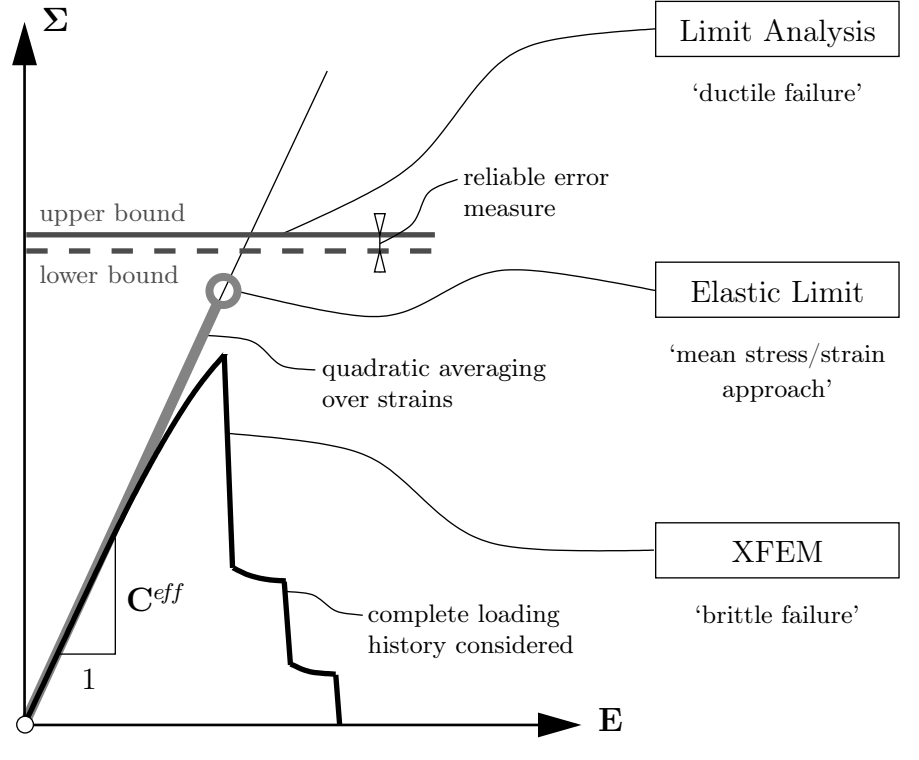

(a)
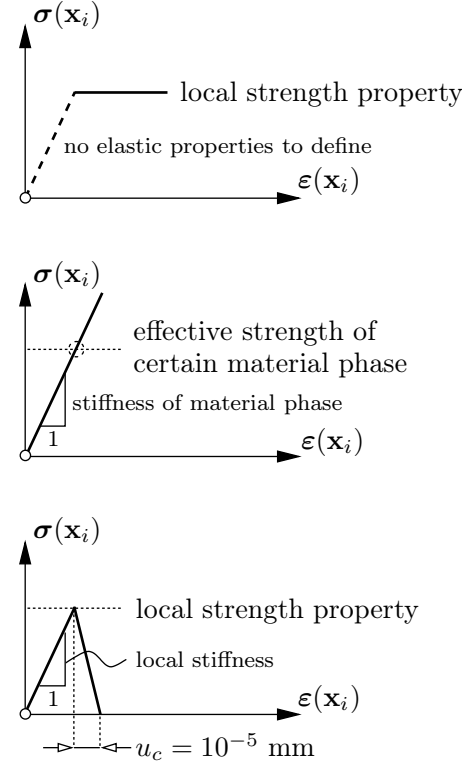

(b)

Figure 3: Characteristics and qualitative representation of the three methods considered: (a) effective/macroscopic stress-strain response and (b) microscopic stress-strain relationships.

The most obvious way to deal with this complex problem, with respect to morphological as well as material behaviour aspects, is to apply the conventional 2D or $3 \mathrm{D}$ finite element method. This has been done by the authors recently, see [1]. Therein, XFEM was applied successfully to representative wood unit cells, delivering effective stress states which cause the initiation of a crack, and simultaneously giving the corresponding effective crack direction. This method is again applied in the present work, leading now to effective strength predictions at two different scales of observation. Nevertheless, even if XFEM, especially in combination with multi-surface plasticity criteria, is a powerful tool allowing for a very realistic description of complex failure mechanisms, its high computational cost and the overall complexity of the approach make 
its use within a multiscale approach rather tedious.

For this reason, additional focus is given to the numerical LA approach and its applicability to microstructures of wood. This method exclusively concentrates on the time instant of failure, and delivers lower and upper bounds for the ultimate strength of the considered material structure. Compared with conventional finite element approaches, where the complete load history has to be considered and, in order to predict the correct failure mechanisms, proper regularisation techniques must be used, the LA approach is much more stable and efficient. Moreover, it leads to rigorous bounds on the material strength, and thus gives a reliable error measure for the prediction. The completely different nature of this so-called direct method, compared with XFEM, becomes obvious in Figure 3.

Of course, the efficiency of LA results from the strict assumptions on which this method is based. These include an associated plastic flow rule and ideal plastic (ductile) behaviour of the considered material. Although these two assumptions are not entirely correct for wood, the first is generally made due to a lack of information about the non-associativity, while the second does not exclude the possibility that good strength predictions can also be obtained for strain-softening structures. In [20] it is stated: "A structure does not need to exhibit perfect plasticity for the theoretical plastic collapse load based on the peak yield stress of each component to be approached closely. Rather, it is necessary that, at the point when a collapse mechanism forms under a particular loading, all those regions within the structure which are undergoing straining lie very close to the peak yield stress which they can achieve." Wood certainly has the ductile potential to 'activate' the strength of many points along a potential crack surface before brittle failure occurs. It therefore seems that LA could be an appropriate method for determining effective strengths, at least for pressure- and shear-dominated loading states.

The EL approach has been chosen as third method. It is based on continuum micromechanical homogenisation schemes $[21,22]$ characterised by linearly averaging stress and strain fields of material phases to obtain the effective stiffness tensor of the corresponding material system. Higher-order strain averages, in combination with an appropriate failure criterion for the failure-pertinent material phase, can then be used to predict effective strengths. These strengths refer to elastic limit states in case of ductile failure, but represent ultimate strengths in the case of brittle failure. For this reason, the EL approach ideally complements the LA approach, and thus an effective multiscale approach delivering comprehensive failure information for clear wood seems to be possible.

The adopted formulations of the three different computational methods, which are subsequently applied to the wood microstructures, are presented in the following subsections.

\subsection{Extended finite element method (XFEM)}

The XFEM, first introduced in [23] and based on the partition of unity method proposed in [24], allows, through targeted enrichment of the approximation possibilities of the displacement field, the description of singularities and weak or strong discontinuities of the considered field quantity. A field $u(\mathbf{x})$, in our case a displacement field, can thereby be approximated by

$$
u(\mathbf{x}) \approx \sum_{i=1}^{N N} \mathbf{N}_{u, i}(\mathbf{x})\left[\mathbf{q}_{u, i}+\sum_{j=1}^{N G} \mathbf{G}_{j}(\mathbf{x}) \mathbf{q}_{e n r, i j}\right]
$$


where $N N$ is the number of nodes of the finite element mesh, $\mathbf{N}_{u, i}$ represents the conventional element interpolation functions obeying the partition-of-unity property, $\sum_{i=1}^{N N E} N_{i}=1$, with $N N E$ as the number of nodes of an element, $\mathbf{q}_{u, i}$ is the displacement vector, and $\mathbf{q}_{e n r, i j}$ contains the additional degrees of freedom of node $i$, representing the amplitude of the $j$ th extension function $\mathbf{G}_{j}(\mathbf{x}) . N G$ is the number of extension functions.

In this work the basic XFEM capability of a commercial finite element package was used, wherein the socalled level set method $[25,26,27]$ is applied to describe the location and geometry of discrete moving cracks. Two level set functions ( $\phi$ and $\psi$ ), which are two almost-orthogonal signed distance functions, are sufficient to describe arbitrary material interfaces and cracks by using conventional finite element interpolation functions:

$$
\phi \approx \sum_{i=1}^{N N} N_{i} \phi_{i} \text { and } \quad \psi \approx \sum_{i=1}^{N N} N_{i} \psi_{i},
$$

where $\phi$ describes the crack surface in a 3D body and $\psi$ is used to describe the crack front. By combining these two level set functions, an arbitrary evolving crack can be described implicitly as follows (cf. Figure 4):

$$
\left\{\begin{array}{llll}
\text { for all } \mathbf{x} \in \text { crack surface, } & \phi(\mathbf{x})=0 & \text { and } & \psi(\mathbf{x})<0 \\
\text { for all } \mathbf{x} \in \text { crack tip, } & \phi(\mathbf{x})=0 & \text { and } & \psi(\mathbf{x})=0
\end{array}\right.
$$

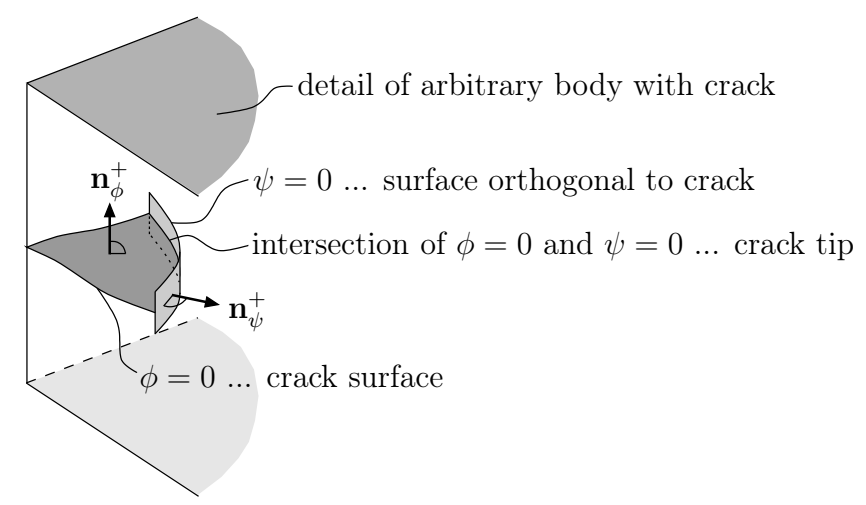

Figure 4: Representation of the two iso-zero level sets $\phi$ and $\psi$ defining crack geometry and location in the XFEM approach.

Commonly, within the XFEM, singularities caused by crack tips are modelled with extension functions derived in the framework of linear elastic fracture mechanics [23]. For elements that are completely cut by a crack, the corresponding nodes are enriched by sign or Heaviside functions. In the present work, for describing cracks (strong discontinuities) only the modified Heaviside function [26] is used, thus

$$
\mathbf{G}_{j}(\mathbf{x})=\mathrm{H}(\phi(\mathbf{x}))=\left\{\begin{array}{ll}
-1 & : \phi(\mathbf{x})<0 \\
1 & : \phi(\mathbf{x}) \geq 0
\end{array} .\right.
$$

The simulation of moving cracks is performed using the phantom node method developed in [28] and based on an approach shown in [29]. Phantom nodes are superimposed on the original nodes, which are 
completely tied to the corresponding original nodes for uncut elements. If the element becomes fully cracked, it is represented by two superimposed elements with a combination of phantom and original nodes, which are no longer constrained to each other. The behaviour of the cracked surfaces can now be described by the same methods that are used for cohesive joint elements. The element-by-element propagation of a crack has been successfully used in [28] for several simulations of crack propagation, with only minor mesh dependency. They also noted that, although the crack speed might be overestimated by the element-byelement propagation of a crack, its propagation path is usually in reasonable agreement with experiments.

After crack initiation, perfect brittle damage evolution is assumed, meaning that the transferable stresses drop almost immediately to zero (the displacement from crack initiation to stress-free crack surfaces is set to $10^{-5} \mathrm{~mm}$, see Figure $3(\mathrm{~b})$ ). To permit subsequent stress transfer over crack surfaces, a node-to-surface contact formulation with finite sliding is used for fully cracked elements.

\subsection{Limit analysis (LA)}

As opposed to conventional finite element methods, XFEM included, LA is a 'direct method', focusing only on the moment of impending plastic collapse. All deformation up to this point is assumed to be of the same order of magnitude as the elastic deformation, so that the influence of geometry changes on the collapse load can be neglected. In the state of impending plastic collapse, all elastic strain rates in the considered body vanish and it behaves like a rigid-plastic rather than an elastic-plastic body. For this reason, only the strength behaviour of each material phase is required, and the need to specify deformation properties (which are often difficult to determine) can be avoided. This argument was first presented by Drucker, Greenberg and Prager [30], together with the first complete formulations of both the lower bound and upper bound theorems of limit analysis [31], which can be stated informally as follows [32]: "The loads that are in equilibrium with a stress field that nowhere violates the yield criterion do not exceed the collapse loads, while the loads that do positive work on a kinematically admissible velocity field at a rate equal to the total plastic dissipation are at least equal to the collapse loads." The required key assumption behind these two theorems is the principle of maximum plastic dissipation, which requires a perfectly plastic material with a convex yield surface and associated plastic flow (normality of incremental plastic strain rates to the yield surface). If the loads resulting from the two theorems are equal to each other, then the true collapse load has been determined. If not, rigorous lower and upper bounds on the exact solution are provided, giving an immediate error indication for the result. Design approaches based on limit analysis represent very efficient methods with a reliability that is often sufficient for many civil and mechanical engineering problems such as structural design and safety assessment, and geotechnical capacity calculations.

In the following, numerical formulations of the bound theorems suitable for application to $2 \mathrm{D}$ wood microstructures will be presented.

Numerical upper bound formulation.

An intuitive statement of the upper bound theorem could read: The considered material structure will collapse under a given loading if an admissible velocity field can be found forming a failure mechanism dissipating less power than the rate of work of the given loading. A velocity field is considered to be 
admissible when it obeys, in every point of the material structure, the associated flow rule

$$
\dot{\varepsilon}=\dot{\lambda} \frac{\partial f}{\partial \boldsymbol{\sigma}} \quad \text { with } \quad \dot{\lambda} \geq 0 .
$$

Here $f$ denotes a convex yield function specified in terms of the Cauchy stress tensor $\sigma, \dot{\lambda}$ is the plastic multiplier and $\dot{\boldsymbol{\varepsilon}}$ represents the strain rate tensor, which is classically linked to the velocity vector $\dot{\mathbf{u}}$, reading

$$
\dot{\varepsilon}_{i j}=\frac{1}{2}\left(\dot{u}_{i, j}+\dot{u}_{j, i}\right)=\mathbf{L}_{u} \dot{\mathbf{u}},
$$

where $\mathbf{L}_{u} \in \mathfrak{R}^{3 \times 2}$ (in 2D) denotes the common differential operator mapping deformations onto strains. In the present work, we are only considering yield functions $f$ that can be written down in the following general quadratic form:

$$
f=\mathbf{F}^{\top} \boldsymbol{\sigma}+\boldsymbol{\sigma}^{\top} \mathbf{P} \boldsymbol{\sigma} \leq 1
$$

where $\mathbf{F} \in \mathfrak{R}^{3}$ and $\mathbf{P} \in \mathfrak{R}^{3 \times 3}$ (in 2D) contain coefficients linked to the strength parameters of the considered material phase. When the symmetric matrix $\mathbf{P}$ is positive definite or semidefinite, the solution set of Equation (7) is an ellipsoid, with the square roots of the eigenvalues of $\mathbf{P}$ giving the semi-axis lengths. It can then be decomposed as $\mathbf{P}=\mathbf{D}^{\top} \mathbf{D}$ with $\mathbf{D} \in \mathfrak{R}^{3 \times 3}$, and Equation (7) can be reformulated into an affine transformation and a cone constraint:

$$
\begin{aligned}
& \mathbf{s}=\mathbf{a}+\mathbf{B} \boldsymbol{\sigma} \\
& \mathbf{s} \in \mathcal{C}
\end{aligned}
$$

with

$$
\mathbf{a}=\left(\begin{array}{lllll}
1 & 0 & 0 & 0 & 0
\end{array}\right)^{\boldsymbol{\top}} \in \mathfrak{R}^{5} \quad \text { and } \quad \mathbf{B}=\left(\begin{array}{c}
-\mathbf{F}^{\mathbf{\top}} / 2 \\
\mathbf{D} \\
\mathbf{F}^{\boldsymbol{\top}} / 2
\end{array}\right) \in \mathfrak{R}^{5 \times 3}
$$

and $\mathcal{C}$ denoting a second-order cone ${ }^{1}$.

Considering Equation (8) and following the derivations in [34], the flow rule in Equation (5) may alternatively be written as

$$
\dot{\varepsilon}=\dot{\lambda} \frac{\partial f}{\partial \boldsymbol{\sigma}}=-\mathbf{B}^{\top} \mathbf{y}
$$

where $\mathbf{y} \in \mathfrak{R}^{5}$ contains terms related to the strain rates.

\footnotetext{
${ }^{1}$ A set $\mathcal{C}$ is called a cone if $\forall \mathbf{x} \in \mathcal{C}$ and $\lambda \geq 0, \lambda \mathbf{x} \in \mathcal{C}$. Examples of such cones are

- the nonnegative orthant

$$
\mathcal{C}=\mathfrak{R}_{+}=\{x: x \geq 0\}
$$
}

- the second-order (or ice-cream) cone

$$
\mathcal{C}=\left\{\mathbf{x} \in \mathfrak{R}^{m}: \sqrt{\sum_{i=2}^{m} x_{i}^{2}} \leq x_{1}, x_{1} \geq 0\right\}
$$

- and the rotated quadratic cone

$$
\mathcal{C}=\left\{\mathbf{x} \in \mathfrak{R}^{m}: \sum_{i=3}^{m} x_{i}^{2} \leq 2 x_{1} x_{2}, x_{1} \geq 0, x_{2} \geq 0\right\}
$$


Finally, in accordance with [34], the upper bound optimisation problem takes the form

$$
\begin{array}{rlr}
\min W_{\text {int }} & =\int_{V} \mathbf{a}^{\top} \mathbf{y} \mathrm{d} V & \\
\text { s.t. } & \mathbf{B}^{\top} \mathbf{y}+\mathbf{L}_{u} \dot{\mathbf{u}}=\mathbf{0} & \text { in } V \\
& \mathbf{y} \in \mathcal{C}^{\star} & \text { in } V \\
& \mathbf{q}^{\top} \dot{\mathbf{u}}=1 &
\end{array}
$$

where $\mathcal{C}^{\star}$ is the dual cone to $\mathcal{C}$ and $\mathbf{q}$ is the vector of equivalent nodal loads arising from the surface tractions t. In this work, since we want to apply the formulation to a unit cell, the last line in Equation (14), scaling the external work rate to 1 , is replaced by periodic boundary conditions formulated in terms of the surface velocities, reading

$$
\dot{\mathbf{u}}^{+}=\dot{\mathbf{u}}^{-}+\dot{\mathbf{E}}\left(\mathbf{x}^{+}-\mathbf{x}^{-}\right) \quad \text { on } \Omega
$$

where $\dot{\mathbf{u}}^{+}$is the velocity vector at the surface node $\mathbf{x}^{+}$, associated with the surface part $\partial \Omega^{+}$, and $\dot{\mathbf{u}}^{-}$is the velocity vector at the surface node $\mathbf{x}^{-}$, associated with the opposite surface part $\partial \Omega^{-}$. The pairs of opposite surfaces $\partial \Omega^{-}$and $\partial \Omega^{+}$are given in Figure 6 , for the unit cell at the clear wood scale as well as for the two unit cells at the annual ring scale. $\dot{\mathbf{E}}$ denotes the effective/macroscopic strain rate tensor of the considered unit cell, and is linked to the microscopic strain rate field as follows:

$$
\dot{\mathbf{E}}=\frac{1}{V} \int_{V} \dot{\varepsilon}(\mathbf{x}) \mathrm{d} V
$$

Since the vector $\mathbf{y}$, and thus the internal work rate in Equation (14) can be written explicitly only for selected yield criteria, the dual form of the optimisation problem in Equation (14) is considered subsequently, reading

$$
\begin{array}{rll}
\max W_{e x t} & =\int_{\partial \Omega^{+}} \dot{\mathbf{u}}^{+\mathbf{T}} \mathbf{t}^{+} \mathrm{d} S+\int_{\partial \Omega^{-}} \dot{\mathbf{u}}^{-\boldsymbol{T}} \mathbf{t}^{-} \mathrm{d} S & =\int_{\partial \Omega^{+}}\left(\dot{\mathbf{E}}\left(\mathbf{x}^{+}-\mathbf{x}^{-}\right)\right)^{\top} \mathbf{t}^{+} \mathrm{d} S \\
\text { s.t. } & \mathbf{L}_{u}^{\top} \boldsymbol{\sigma} \mathrm{d} V-\left(\mathbf{t}^{+}-\mathbf{t}^{-}\right) \mathrm{d} \Omega=\mathbf{0} & \text { in } V \\
& \mathbf{s}=\mathbf{a}+\mathbf{B} \boldsymbol{\sigma} & \text { in } V \\
& \mathbf{s} \in \mathcal{C} & \text { in } V
\end{array}
$$

where the dual variables $\boldsymbol{\sigma}$ represent stress-like quantities, $\mathrm{d} V$ the associated volume on which $\boldsymbol{\sigma}$ acts, and $\mathrm{d} \Omega$ the surface part relevant to either $\mathbf{t}^{+}$or $\mathbf{t}^{-}$.

The finite element discretisation for the upper bound problem uses the triangular elements depicted in Figure 5 (left side). Quadratic shape functions are used to approximate the velocity field, as proposed in [33], resulting in a linear variation of strain rate within the element. If the vertices of the element are used to enforce the flow rule and evaluate the plastic dissipation, the solutions obtained are strict upper bounds on the exact collapse load $[35,36]$. In the case of unstructured meshes, these linear strain rate elements typically show better performance than the often-used constant strain rate elements with velocity discontinuities $[35,36]$.

Finally, the discretised form of the dual upper bound optimisation problem in Equation (17) can be solved efficiently by an interior point method. In this work, the algorithm outlined in [37], which has been implemented in the optimisation software MOSEK [38], was used.

Numerical lower bound formulation.

An intuitive statement of the lower bound theorem could read: A body will not collapse under a certain 

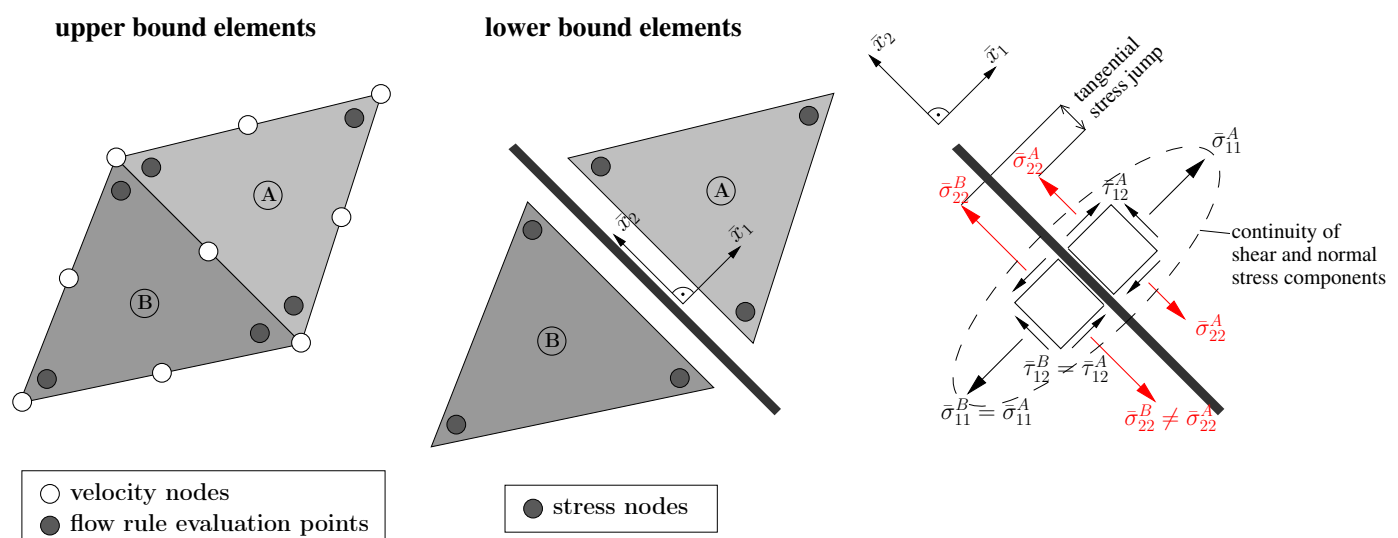

$\bigcirc$ velocity nodes

flow rule evaluation points

Figure 5: Finite elements used in the upper and lower bound LA formulations.

loading if an admissible stress field can be found that is in equilibrium with a loading greater than the considered loading. A stress field is considered to be admissible when it does not violate the yield criterion, according to Equation (7) or (8), at any point of the material structure.

The optimal lower bound solution can be found by solving the following optimisation problem:

$$
\begin{array}{lll}
\max & \alpha & \\
\text { s.t. } & \mathbf{L}_{\sigma} \boldsymbol{\sigma}=\mathbf{0} & \text { in } V \\
& \mathbf{t}^{+}+\mathbf{t}^{-}=\mathbf{L}_{t}\left(\boldsymbol{\sigma}^{+}+\boldsymbol{\sigma}^{-}\right)=\mathbf{0} & \text { on } \Omega \\
& \frac{1}{\Omega^{+}} \int_{\Omega^{+}} \mathbf{L}_{t} \boldsymbol{\sigma}^{+} \mathrm{d} S=\alpha \mathbf{T}^{+} & \text {on } \Omega^{+} \\
& \mathbf{s}=\mathbf{a}+\mathbf{B} \boldsymbol{\sigma} & \text { in } V \\
& \mathbf{s} \in \mathcal{C} & \text { in } V
\end{array}
$$

In the first constraint, which enforces equilibrium within the body $V, \mathbf{L}_{\sigma}$ denotes the divergence operator. The second constraint represents the static periodic boundary conditions, necessary when applying this formulation to unit cells, with $\mathbf{t}^{+}$as the traction at the surface node $\mathbf{x}^{+}$, associated with the surface part $\partial \Omega^{+}$, and $\mathbf{t}^{-}$as the traction at the surface node $\mathbf{x}^{-}$, associated with the opposite surface part $\partial \Omega^{-}$. The matrix $\mathbf{L}_{t}$ depends on the outward normal vector $\mathbf{n}$ of the surface $\Omega$ and connects the stresses $\boldsymbol{\sigma}^{+}$and $\boldsymbol{\sigma}^{-}$at the surface to the corresponding tractions. Finally, through $\mathbf{T}$ the effective traction loading at the surface of the considered unit cell is specified, which is self-equilibrating due to the simultaneously enforced periodic boundary conditions.

To obtain a discretised version of the lower bound optimisation problem in Equation (18), the stress field is approximated by a piecewise linear variation using triangular finite elements as shown in Figure 5 (middle). Each element has its own set of three internal stress evaluation nodes, thus multiple nodes from adjacent elements may share the same coordinates. At the resulting stress discontinuities between elements, only the continuity of normal and shear stress components needs to be enforced; a jump in the stress component parallel to the discontinuity is allowed (as illustrated in Figure 5, right side).

Finally, the discretised form of the lower bound optimisation problem can be solved in a similar way to the dual upper bound problem, using the optimisation software MOSEK [38]. 


\subsection{Elastic limit (EL) approach}

Unlike the XFEM and LA approaches described before, the EL approach used within this work is solely based on linking the effective stiffness properties of the considered (loaded) material with the stiffness properties of the underlying material phases. Therefore, depending on the observation scale, either continuum micromechanical schemes [21] or analytical solutions for unit cell structures [39] are employed. Finally, effective stress states of the loaded material structure can be directly related to microscopic strains in an arbitrary material phase. Under the assumption that failure of a certain material phase is responsible for failure of the whole material structure, critical strain states in such a phase can be identified and, subsequently, effective failure stress states of the whole material structure are obtained.

\section{Continuum micromechanics.}

Within the framework of continuum micromechanics, a material is seen as a micro-heterogeneous body filling a macro-homogeneous representative volume element (RVE) at different scales of observation. The microstructure within such an RVE can be described by so-called material phases, representing quasihomogeneous subdomains with known physical characteristics such as volume fractions, characteristic shapes, and mechanical properties. The size of the inhomogeneities defined by the material phases has to be significantly smaller than the characteristic length of the RVE, and the size of the RVE again has to be smaller than the characteristic dimension of the structure built up by the material, or the RVE representing the next higher scale of observation, respectively. In such a way multiscale homogenisation schemes are set up.

In continuum micromechanics, the effective stiffness $\mathbf{C}^{\text {eff }}$ of an RVE can be linked to its microstructure according to [21, 40], based on the solutions in [41, 42], as follows [3]:

$$
\begin{aligned}
\mathbf{C}^{e f f} & =\sum_{i=1}^{N} f_{i} \mathbf{c}_{i}: \mathbf{A}_{i} \quad \text { with } \\
\mathbf{A}_{i} & =\left[\mathbf{I}+\mathbf{P}_{i}^{0}:\left(\mathbf{c}_{i}-\mathbf{C}^{0}\right)\right]^{-1}:\left\{\sum_{j=1}^{N} f_{j}\left[\mathbf{I}+\mathbf{P}_{j}^{0}:\left(\mathbf{c}_{j}-\mathbf{C}^{0}\right)\right]^{-1}\right\}^{-1},
\end{aligned}
$$

where $\mathbf{c}_{i}$ and $f_{i}$ denote the elastic stiffness and the volume fraction of phase $i$ of total $N$ material phases, respectively, $\mathbf{A}_{i}$ is the concentration tensor of phase $i$ and $\mathbf{I}$ is the fourth-order identity tensor. $\mathbf{C}^{\text {eff }}$ relates effective/macroscopic RVE-related stresses $\boldsymbol{\Sigma}$ to corresponding strains $\mathbf{E}$, while $\mathbf{c}_{i}$ relates (average) phase stresses $\boldsymbol{\sigma}_{i}=\left(1 / V_{i}\right) \int_{V_{i}} \boldsymbol{\sigma}(\mathbf{x}) d V$ to (average) phase strains $\varepsilon_{i}=\left(1 / V_{i}\right) \int_{V_{i}} \varepsilon(\mathbf{x}) d V$. x is the location vector for positions inside the RVE, $\boldsymbol{\sigma}(\mathbf{x})$ and $\boldsymbol{\varepsilon}(\mathbf{x})$ are the stress and strain fields inside the material phases, and $V_{i}$ is the volume of phase domain $i$ inside the considered RVE. The fourth-order tensor $\mathbf{P}_{i}^{0}$ accounts for the characteristic shape of phase $i$ in a matrix with stiffness $\mathbf{C}^{0}$. The choice of this stiffness describes the interactions between phases. If $\mathbf{C}^{0}$ describes one of the phase stiffnesses, a material with a contiguous matrix with inclusions is represented and Equation (19) renders the Mori-Tanaka scheme [40], while for $\mathbf{C}^{0}=\mathbf{C}^{e f f}$, a dispersed arrangement of the phases is represented and Equation (19) reflects the self-consistent scheme.

\section{Unit cell homogenisation.}

If the material microstructure is characterised by the spatial variation of physical quantities which can be represented by a combination of local fluctuations at the level of the elementary cell and a spatial repetition of this elementary cell, a unit cell homogenisation scheme may be employed. The honeycomb structure inherent to wood, presented later, represents such a material microstructure, which can be built up/approximated 
using identical basic repetitive units. Moreover, the length of these cells is two orders of magnitude larger than their cross-sectional dimensions, justifying the assumption of an infinite extension of the unit cells in the longitudinal direction (i.e., conditions of plane strain). Thus, the unit cell homogenisation methods proposed in [39, 43] can be applied.

Thereby, the unit cell is subjected to periodic, symmetry, or antisymmetry displacement boundary conditions such that the spatial averages of the corresponding microscopic strains $\varepsilon(\mathbf{x})$ are equal to the macroscopic strains $\mathbf{E}$ related to the cellular material. Linking these macroscopic strains to the spatial average of the periodic microstresses they induce, i.e., to the macroscopic stresses $\boldsymbol{\Sigma}$, yields the homogenised effective stiffness $\mathbf{C}^{e f f}$ of the cellular material. The effective stiffness expressions, as functions of the unit cell characteristics, and the original application of this method to wood can be found in [44]. In contrast to continuum micromechanics, potential plate-like bending and shear deformations of the cell walls, building up the material structure, can be taken into account with this homogenisation method.

\section{Elastic Limit estimates.}

Both of the described methods, continuum micromechanics as well as unit cell homogenisation, can be used, either alone or in combination, to determine the effective stiffness $\mathbf{C}^{e f f}$ of a material structure as a function of its microstructural characteristics.

Let us now assume that the intensity of shear loading of the weakest phase is responsible for failure (an elastic limit state) of our considered material structure. Then, we would need an appropriate stress or strain measure for this material phase and a corresponding failure criterion. In [22] such a strain measure is proposed, in fact, quadratic strain averages (second-order moments) $\bar{\varepsilon}_{i}^{d}$ are assumed to appropriately represent (failure-causing) local strain peaks in a material phase $i$, reading

$$
\bar{\varepsilon}_{i}^{d}=\sqrt{\frac{1}{V_{i}} \int_{V_{i}} \frac{1}{2} \varepsilon^{d}(\mathbf{x}): \varepsilon^{d}(\mathbf{x}) d V,}
$$

where $\varepsilon^{d}(\mathbf{x})$ denotes the deviatoric strain field, defined as $\varepsilon^{d}(\mathbf{x})=\varepsilon-(\operatorname{tr} \varepsilon(\mathbf{x}) / 3) \mathbf{I}$. According to Hill's lemma [45], the following relationship between microscopic and macroscopic quantities, representing the energy density $\mathcal{E}$, is valid:

$$
\mathcal{E}=\frac{1}{V_{i}} \int_{V_{i}} \frac{1}{2} \varepsilon(\mathbf{x}): \mathbf{c}_{i}: \varepsilon(\mathbf{x}) d V=\frac{1}{2} \mathbf{E}: \mathbf{C}^{e f f}: \mathbf{E},
$$

where $\mathbf{c}_{i}=3 k_{i} \mathbf{J}+2 \mu_{i} \mathbf{K}$ is the isotropic stiffness of material phase $i$, with bulk modulus $k_{i}$ and shear modulus $\mu_{i}$, and with $\mathbf{J}$ and $\mathbf{K}=\mathbf{I}-\mathbf{J}$ being the volumetric and deviatoric parts of the fourth-order identity tensor I. With $\partial \mathbf{c}_{i} / \partial \mu_{i}=2 \mathbf{K}$, the derivatives of expressions (21) with respect to the shear modulus $\mu_{w}$ of the weakest phase (with a volume content of $f_{w}$ ) read

$$
\frac{\partial \mathcal{E}}{\partial \mu_{w}}=2 f_{w}\left(\overline{\bar{\varepsilon}}_{w}^{d}\right)^{2}=\frac{1}{2} \mathbf{E}: \frac{\partial \mathbf{C}^{e f f}}{\partial \mu_{w}}: \mathbf{E},
$$

finally providing an expression for the quadratic strain average of the weakest phase as a function of the effective stiffness and the effective strain, reading

$$
\overline{\bar{\varepsilon}}_{w}^{d}=\sqrt{\frac{1}{4 f_{w}} \mathbf{E}: \frac{\partial \mathbf{C}^{e f f}}{\partial \mu_{i}}: \mathbf{E} .}
$$


In turn, the effective strain is related to the effective stress according to $\boldsymbol{\Sigma}=\mathbf{C}^{\text {eff }}$ : $\mathbf{E}$. With an appropriate failure criterion for the weakest phase of the considered material structure,

$$
F_{w}\left(\bar{\varepsilon}_{w}^{d}\right)=0,
$$

effective elastic limit stress states $\boldsymbol{\Sigma}^{E L}$ and, subsequently, an effective failure criterion $F\left(\boldsymbol{\Sigma}^{E L}\right)=0$ can be obtained.

\section{Material}

\subsection{Morphology}

The most common wood species used in engineering constructions is spruce, thus, it is a very well investigated material with a large body of experimental data about its microstructural characteristics and the properties of its constituents. For this work, the composition of spruce wood was taken from [46], in which a comprehensive micromechanical model for the elasticity of wood is presented, and many references to experimental data for the wooden microstructure are given. Those data and similar models were also used in further works, e.g., in [44], presenting an improved micromechanical model for the elasticity of wood, in [3], proposing an extension to obtain elastic limit states of wood, and in [47, 48], including a poromechanical approach in the micromechanical model.

Based on the underlying data of these models, appropriate observation scales have been defined in the present work, tailored for predicting the effective strength of clear wood based on its microstructural characteristics. The highest observation scale in this multiscale approach, illustrated in Figure 6, represents the clear wood scale, consisting of earlywood and latewood layers with a typical thickness between 0.3 and $3.5 \mathrm{~mm}$. Considering the stem cross-section of a tree, the earlywood and latewood layers form a pattern of concentric circles around the pith, known as growth rings. $T$ indicates the tangential direction and $R$ the radial direction with respect to these growth rings, while $L$ shows the longitudinal direction (stem direction).

At the next scale of observation in Figure 6, the annual ring scale, the earlywood and latewood layers consist of periodically arranged honeycomb cells, and thus they can be appropriately represented by unit cells. For both the earlywood and latewood layers, two different unit cells have been defined. The less complex ones (upper cells in Figure 6) are used within the analytical EL approach, while the ones consisting of two different layers (lower cells in Figure 6) serve as the basis for the numerical (XFEM and LA) calculations. Both unit cell types represent the same kind of earlywood and latewood layer, only a different representation of the cell wall has been used, accounting for the possibilities of the particular computational method. In reality, the cell wall consists of five different layers (often referred to as the middle lamella, P, S1, S2, and S3 layers). The volumes of the P, S1 and S3 layers are very small compared with the S2 layer and the middle lamella. For this reason, only two layers are distinguished in the unit cell for the numerical approaches: the middle lamella $(\mathrm{ml})$ and a homogenised layer ( $\mathrm{sl}$ ) representing all S layers and the P layer. The analytical unit cell for the EL approach is completely homogeneous, merging the properties of all five layers into one cell wall material $(\mathrm{cw})$.

Each cell wall layer is itself made up of a non-cellulosic matrix (termed polymer network, pn) in which cellulosic fibres (cf) are embedded, extending spirally at an angle MFA (micro fibril angle) towards the cell 


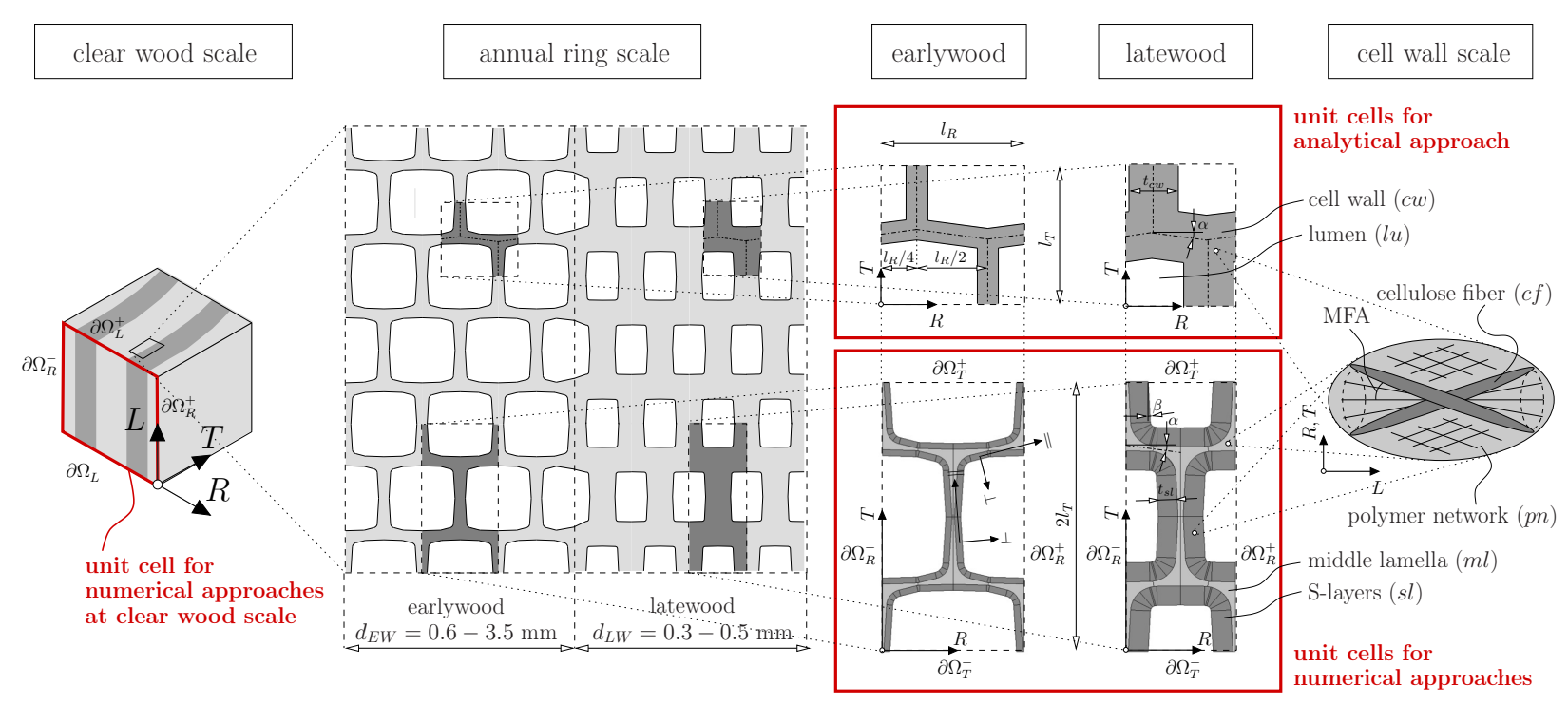

Figure 6: Definition of unit cells used for the numerical approaches (XFEM, LA) and the analytical approach (EL) at annual ring scale and clear wood scale for the considered spruce wood (experimentally investigated in [49]). The 3D unit cells for the XFEM and EL approaches were obtained by simple extrusion of the $2 \mathrm{D}$ cross sections shown.

\subsection{Mechanical behaviour}

To obtain the effective elastic behaviour at the cell wall scale (cf. Figure 6, right side), Equation (19) was applied, with $\mathbf{C}^{0}$ as the isotropic stiffness of the polymer network $\mathbf{c}_{p n}$, and $\mathbf{c}_{1}$ as the anisotropic stiffness of the cellulosic fibres $\mathbf{c}_{c f}$. The stiffness tensors $\mathbf{c}_{p n}$ and $\mathbf{c}_{c f}$ were calculated according to the model in [48], yielding

$$
\begin{aligned}
\mathbf{c}_{c f} & =\left[\begin{array}{cccccc}
16779 & 5626 & 2390 & & & \\
& 16779 & 2390 & & & \\
& & 113123 & & & \\
& & & 7532 & & \\
& \text { sym. } & & & 7532 & \\
& & & & & 11153
\end{array}\right] \text { and } \\
\mathbf{c}_{p n} & =\left[\begin{array}{lccccc}
4011 & 1496 & 1496 & & & \\
& 4011 & 1496 & & & \\
& & 4011 & & & \\
& & & 2515 & & \\
& \text { sym. } & & 2515 & \\
& & & & & 2515
\end{array}\right][\mathrm{MPa}] .
\end{aligned}
$$

axis. The volume contents of these different material phases, at the different observation scales, can be in Table 1

The parameters defining the geometries of the unit cells in Figure 6 are given in Table 2 .

The cellulosic fibres are modelled as cylindrical inclusions, defined through the concentration tensor $\mathbf{P}_{c f}^{0}$, which can be found in [21]. The volume content of the cellulosic fibre phase $f_{c f}$ is given in Table 1 . The 


\begin{tabular}{|c|c|c|c|c|}
\cline { 2 - 5 } \multicolumn{1}{c|}{} & \multicolumn{2}{c|}{ cell wall scale } & \multicolumn{2}{c|}{ unit cells } \\
\cline { 2 - 5 } & middle lamella $(m l)$ & S-layers $(s l)$ & EW & LW \\
\hline \multirow{3}{*}{ numerical approaches } & $f_{p n}=0.9$ & $f_{p n}=0.6$ & $f_{m l}=0.36$ & $f_{m l}=0.23$ \\
\cline { 2 - 5 } & $f_{c f}=0.1$ & $f_{c f}=0.4$ & $f_{s l}=0.64$ & $f_{s l}=0.77$ \\
\hline \multirow{3}{*}{ all approaches } & \multicolumn{3}{|c|}{ annual ring scale } \\
\cline { 2 - 5 } & \multicolumn{2}{|c|}{ unit cells } & EW & LW \\
\cline { 2 - 5 } & $f_{p n}=0.71$ & $f_{p n}=0.67$ & $f_{c w}=0.24$ & $f_{c w}=0.61$ \\
\hline & $f_{c f}=0.29$ & $f_{c f}=0.33$ & $f_{l u}=0.76$ & $f_{l u}=0.39$ \\
\hline
\end{tabular}

Table 1: Volume content of different material phases at different scales of observation for a typical spruce wood as investigated in [49] and modelled in [46]. EW = earlywood, LW = latewood. Indices are explained in Figure 6.

\begin{tabular}{|c|c|c|c|c|c|c|c|}
\hline & & \multicolumn{2}{|c|}{ shape of unit cells } & \multicolumn{4}{|c|}{ cell wall geometry } \\
\hline & & $l_{R}[\mu \mathrm{m}]$ & $l_{T}[\mu \mathrm{m}]$ & $\alpha\left[^{\circ}\right]$ & $\beta\left[^{\circ}\right]$ & $t_{m l}[\mu \mathrm{m}]$ & $t_{s l}[\mu \mathrm{m}]$ \\
\hline \multirow{2}{*}{ 'numerical' unit cell } & EW & 34 & 33 & 8.0 & 3.5 & 1.5 & 1.5 \\
\hline & LW & 26 & 33 & 8.0 & 3.0 & 2.0 & 4.7 \\
\hline & & \multicolumn{2}{|c|}{$l_{T} / l_{R}[-]$} & \multicolumn{2}{|c|}{$\alpha\left[^{\circ}\right]$} & \multicolumn{2}{|c|}{$t_{c w} / l_{R}[-]$} \\
\hline \multirow{2}{*}{ 'analytical' unit cell } & EW & \multicolumn{2}{|c|}{0.97} & \multicolumn{2}{|c|}{8.0} & \multicolumn{2}{|c|}{0.13} \\
\hline & LW & \multicolumn{2}{|c|}{1.27} & \multicolumn{2}{|c|}{8.0} & \multicolumn{2}{|c|}{0.43} \\
\hline
\end{tabular}

Table 2: Parameters defining the geometry and dimensions of the earlywood (EW) and latewood (LW) unit cells illustrated in Figure 6, for a typical spruce wood as investigated in [49] and modelled in [46]. 
resulting effective cell wall layer stiffnesses define the elastic behaviour of the wood unit cells at the annual ring scale, and thus serve as input to the XFEM simulations as well as to the unit cell homogenisation within the EL approach. The elegance of this continuum micromechanical approach is that it efficiently relates the elastic cell wall layer behaviour to the behaviour of its microstructural building blocks, like amorphous cellulose, hemicellulose, lignin, water, crystalline cellulose, and so on. The mechanical properties of these phases are the same for different wood species, and thus, the input parameters for the presented multiscale concept can easily be adapted for different species of wood. No elastic material properties are required for the LA approaches.

The strength behaviour assigned to the wood cell structures is explained in the following. In the course of the EL approach in [48], lignin is assumed to be the weakest phase in the wood material structure, failing in shear once a certain threshold is achieved [50]. Thus, an appropriate failure criterion for use in Equation (24) reads

$$
F_{w}\left(\overline{\bar{\varepsilon}}_{w}^{d}\right) \widehat{=} F_{\text {lignin }}\left(\overline{\bar{\varepsilon}}_{\text {lignin }}^{d}\right)=\left(2 \mu_{\text {lignin }} \overline{\bar{\varepsilon}}_{\text {lignin }}^{d}\right)^{2}-\left(\tau_{\text {lignin }}\right)^{2},
$$

where $\tau_{\text {lignin }}$ denotes the lignin shear strength, assumed to be $14.3 \mathrm{MPa}$ according to $[48,1]$. Since it can be assumed that failure of the middle lamella is primarily caused by failure of its main constituent lignin, the shear strength of lignin (and the Von Mises-type failure criterion in Equation (27)) was also assigned to the middle lamella of the unit cells for the numerical approaches, $\tau_{m l} \approx \tau_{\text {lignin }}=14.3 \mathrm{MPa}$ (see also Table 3 ).

Recently, it has also been shown in [51] that isolated pieces of the cell wall (so-called micropillars) can be tested in compression and, subsequently, the shear yield stress of the polymer matrix in wood can be estimated. The obtained values in [51] agree very well with the values used in this work. This current trend of micro-compression and tensile tests at this scales of observation will provide a wide range of reliable strength input parameters for multiscale concepts, as presented in this paper, in future.

\begin{tabular}{|l|c|c|c|c|c|}
\cline { 2 - 6 } \multicolumn{1}{c|}{} & middle lamella $(m l)$ & \multicolumn{4}{c|}{ S-layers $(s l)$} \\
\cline { 2 - 6 } \multicolumn{1}{c|}{} & $\tau_{m l}[\mathrm{MPa}]$ & $\sigma_{\|}^{s l}[\mathrm{MPa}]$ & $\sigma_{\perp}^{s l}[\mathrm{MPa}]$ & $\tau_{\| \perp}^{s l}=\tau_{\| L}^{s l}[\mathrm{MPa}]$ & $\sigma_{L}^{s l}[\mathrm{MPa}]$ \\
\hline XFEM & 14.3 & 75 & $\infty$ & 43 & 175 \\
\hline Limit Analysis & 14.3 & 75 & $150 / \infty$ & 43 & - \\
\hline Elastic Limit & $\tau_{m l} \approx \tau_{\text {lignin }}=14.3$ & $\infty$ & $\infty$ & $\infty$ & $\infty$ \\
\hline
\end{tabular}

Table 3: Strength values assigned to middle lamella and S-layers of the earlywood and latewood unit cells, for the three different methods. Normal failure stresses $\sigma^{s l}$ represent absolute values, valid for compression as well as tension. Indices are explained in Figure 6.

In contrast to the EL approach, for the numerical approaches the strength behaviour of the explicitly modelled S-layer also needs to be defined. This layer resembles a unidirectional fibre-reinforced composite, a material for which several failure criteria have been developed. Following Puck's failure criterion [52], which identifies fibre failure and inter-fibre failure in a unidirectional composite, the following three criteria have 
been assigned to the S-layers, indicating crack initiation within the XFEM approach [1]

$$
\begin{aligned}
f_{s l, 1}(\boldsymbol{\sigma}) & \leq \frac{\sigma_{L}}{\sigma_{L}^{s l}}, \\
f_{s l, 2}(\boldsymbol{\sigma}) & \leq \frac{\sigma_{\|}}{\sigma_{\|}^{s l}}, \quad \text { and } \\
f_{s l, 3}(\boldsymbol{\sigma}) & \leq\left(\frac{\tau_{\| \perp}}{\tau_{\| \perp}^{s l}}\right)^{2}+\left(\frac{\tau_{\| L}}{\tau_{\| L}^{s l}}\right)^{2} .
\end{aligned}
$$

Equation (28) describes Mode I fibre failure in $L$-direction, whereas Equations (29) and (30) both describe inter-fiber failure, leading to a fracture plane with its normal pointing toward the local $\|$-direction (see Figure 6 for definitions of the symbols $\|$ and $\perp$, which respectively denote the local parallel and perpendicular directions in the 'numerical' unit cell). The corresponding tensile and shear strength values were chosen in the same way as in [1], and are given in Table 3.

For strength definition in the LA approach, two different strategies were followed. On one hand, the in-plane failure criteria as used in the XFEM approach, Equations (29) to (30), were assigned to the S-layers, and on the other hand, a single 2D anisotropic Tsai-Wu yield criterion [53] was used, reading

$$
f_{s l, T W}(\boldsymbol{\sigma})=\frac{\sigma_{\perp}^{2}}{\left(\sigma_{\perp}^{s l}\right)^{2}}+\frac{\sigma_{\|}^{2}}{\left(\sigma_{\|}^{s l}\right)^{2}}+\frac{\tau_{\| \perp}^{2}}{\left(\tau_{\| \perp}^{s l}\right)^{2}} \leq 1 .
$$

Both sets of failure criteria gave almost identical results, but due to the slightly lower numerical effort of the Tsai-Wu criterion, it is used for all LA simulations presented in this paper. All the criteria from Equations (29) to (31) can be cast in a general quadratic form according to Equation (7), and thus can be expressed as second-order cone constraints, as required for the LA optimisation problems in Equations (17) and (18).

For the annual ring scale, no experimentally-obtained strength values are available for assessing the computational results. For this reason, all three of the selected methods were also used to obtain effective strength values at the clear wood scale. No extension of the EL approach was necessary to achieve this; only the geometry of the wood unit cell was changed to represent a mean value of earlywood and latewood and, in this manner, directly give approximate failure estimates for clear wood. In accordance with Table 2, the parameters defining the 'analytical' unit cell geometry for the clear wood yield $l_{T} / l_{R}=1.12, \alpha=8.0^{\circ}$, and $t_{c w} / l_{R}=0.28$. This geometry was adapted to comply with the mean mass density, $\rho_{C W}=0.53 \mathrm{~g} / \mathrm{cm}^{3}$, of the investigated clear wood material. The mass densities represented by the wood unit cells at the annual ring scale are $0.35 \mathrm{~g} / \mathrm{cm}^{3}$ for earlywood and $0.90 \mathrm{~g} / \mathrm{cm}^{3}$ for latewood, with a mean mass density of the cell wall material of $1.45 \mathrm{~g} / \mathrm{cm}^{3}$.

For the numerical approaches at the clear wood scale, a simple unit cell was used as shown in Figure 6 (left side). For these analyses, focus was laid on the $L-R$ plane, the plane for which experimental biaxial testing results are available. At this scale, both annual ring types (earlywood and latewood) are modelled as homogeneous materials, with properties obtained from the modelling results from the scale below. The effective strength values obtained from the 3D XFEM simulations of the earlywood and latewood unit cells can be used to define multiple 3D Tsai-Wu failure surfaces, describing the failure behaviour of the annual rings at the clear wood scale. Thus, instead of the failure criteria (28) to (30) within the XFEM approach, multiple Tsai-Wu failure surfaces indicate crack initiation within the annual rings at clear wood scale. All 
the parameters defining these failure criteria and the corresponding definitions of crack directions can be found in [1]. In the LA approach at clear wood scale, the strength behaviour of each annual ring type is described by a single 2D Tsai-Wu failure surface, defined through the strength values given in Table 4 . The radial strengths $\sigma_{R}$ for the earlywood and latewood were taken from the 2D LA calculations for the

\begin{tabular}{|l|c|c|c|c|c|}
\cline { 2 - 7 } \multicolumn{1}{c|}{} & \multicolumn{5}{c|}{ earlywood (EW) } \\
\cline { 2 - 7 } \multicolumn{1}{c|}{} & failure criterion & $\sigma_{L, \text { ten }}^{E W}[\mathrm{MPa}]$ & $\sigma_{L, \text { comp }}^{E W}[\mathrm{MPa}]$ & $\sigma_{R}^{E W}[\mathrm{MPa}]$ & $\tau_{L R}^{E W}[\mathrm{MPa}]$ \\
\hline \multirow{2}{*}{ XFEM } & mult. Tsai Wu & $(60.0)$ & $(35.4)$ & $(5.3)$ & $(8.6)$ \\
\hline Limit Analysis & Tsai Wu & 60.0 & 35.4 & 5.3 & 8.6 \\
\hline & Von Mises & \multicolumn{5}{c|}{$\tau_{\text {lignin }}=14.3$} \\
\cline { 2 - 7 } & \multicolumn{5}{c|}{ latewood $(\mathbf{L W})$} \\
\cline { 2 - 7 } & failure criterion & $\sigma_{L, t e n}^{L W}[\mathrm{MPa}]$ & $\sigma_{L, c o m p}^{L W}[\mathrm{MPa}]$ & $\sigma_{R}^{L W}[\mathrm{MPa}]$ & $\tau_{L R}^{L W}[\mathrm{MPa}]$ \\
\hline XFEM & mult. Tsai Wu & $(126.0)$ & $(58.0)$ & $(13.0)$ & $(8.6)$ \\
\hline Limit Analysis & Tsai Wu & 126.0 & 58.0 & 13.0 & 8.6 \\
\hline Elastic Limit & Von Mises & \multicolumn{5}{c|}{} \\
\hline
\end{tabular}

Table 4: Strength values for earlywood and latewood layers at the clear wood scale, for the three different methods. $\sigma_{R}^{E W}$ is valid for tension (ten) as well as compression (comp). Indices are explained in Figure 6 .

respective unit cells at the annual ring scale. Since these calculations delivered no strength properties in the $L$-direction, those values were taken from the multi-surface failure criteria obtained by XFEM. That is the reason why the XFEM values in Table 4 are in parentheses, because they describe points of the multi-surface but are not directly linked to Tsai-Wu failure surface parameters. Again, the 2D Tsai-Wu failure criterion in Equation (31) can be cast in a general quadratic form according to Equation (7), and thus, the upper and lower bound LA formulations (Equations (17) and (18)) can also be applied at the clear wood scale.

Finally, each of the three methods described in Section 2 delivers predictions of effective strength for two different scales of observation: the annual ring scale and the clear wood scale. Selected results from all these computations will be presented in the following section.

\section{Calculations and results}

When the strength parameters as described in the previous section were chosen, particular attention was paid to ensure that the results of the different methods remain comparable, meaning that all three methods still describe the same wooden material. Differences in computational results can then be primarily attributed to the respective method, and a valid performance assessment with respect to wood may be issued. In the following section, selected computational results from all three methods are presented and compared with each other, giving insights into their predictive qualities.

By varying the effective (macroscopic) loading situation applied to the unit cell under consideration, either the honeycomb unit cell at the annual ring scale or the layered unit cell at the clear wood scale (see 
Figure 6), effective failure surfaces $F(\boldsymbol{\Sigma})$ for those unit cells can be obtained. Within the XFEM approach, loading is applied through a prescribed displacement field, fulfilling periodicity, at the boundary of the relevant unit cell. The maximum attainable reaction force at the boundary, for a certain load situation, defines the corresponding effective failure stress state $\boldsymbol{\Sigma}^{X F E M}$. Within the upper bound LA approach, the effective strain rate tensor $\dot{\mathbf{E}}$ in Equation (17) is varied and effective failure stress states $\boldsymbol{\Sigma}^{U B}$ are obtained by taking the mean value of the microscopic stresses of the optimal solution. The lower bound LA approach leads to different failure stress states $\boldsymbol{\Sigma}^{L B}$ through variation of the effective traction $\mathbf{T}^{+}$applied in Equation (18). Finally, using the EL approach, effective elastic limit stress states $\boldsymbol{\Sigma}^{E L}$ can easily be obtained as described in the last paragraph of Section 2.3.

\subsection{Annual ring scale}

In Figure 7 the effective failure surfaces obtained for earlywood in the $R$ - $T$ plane are shown, together with selected failure modes obtained with upper bound LA and XFEM. The XFEM failure surface consists of six Tsai-Wu criteria fitted to the numerically-obtained effective stress states $\boldsymbol{\Sigma}^{X F E M}$. The detailed procedure and definition of the failure surfaces shown here can be found in [1]. LA provides both lower and upper bound envelopes for the effective strength, with both surfaces based on 86 calculations ${ }^{2}$. Despite the fairly complex structural characteristics of the unit cell, the achieved bound gaps, defined as the difference between the lower and upper bounds, are very small. For the majority of the loading states this gap is less than $1.5 \%$, and even the worst value is just $3.6 \%$.

As would be expected according to the nature and characteristics of the various methods (see Figure 3), the LA results enclose the results of the XFEM approach. This difference is larger in tension-dominated regions, because there, the difference between brittle (XFEM) and ductile (LA) failure becomes obvious. Nevertheless, compared with the EL approach, the LA method seems to capture the basic failure characteristics quite well and shows a qualitatively similar effective failure behaviour to that delivered by the XFEM approach. A good agreement is also revealed by comparing the related failure modes of LA and XFEM in Figure 7. At this point, it is thus shown for the first time that the application of LA approaches to wood might be reasonable, and taking their great efficiency into account, even very promising. The same cannot be said of the EL estimates for earlywood, which differ strongly from the XFEM and LA results. The cell wall running in the $R$-direction is only slightly inclined, and therefore, structural failure is only achieved when the normal failure stress of the cellulosic fibre-rich S-layers (cf. Table 1) is exceeded, and not simply when the lignin-rich middle lamella fails. Since the EL approach used here considers lignin failure as the indication for structural failure, the elastic limit states highly underestimate the strength in the $R$-direction. A completely different picture emerges in the $T$-direction, where bending and shear loading of the cell wall is predominant, and thus, shear failure of the lignin-rich phases contributes the majority of the dissipated energy at structural failure. In this case, the quadratic strain averages, according to Equations (20) and (23), probably cannot represent the high strain concentrations at the corners of the wooden cell sufficiently, leading to an overestimation of the strength in this direction. To compensate for these weaknesses, a sec-

\footnotetext{
${ }^{2}$ A detailed presentation of all the LA results is beyond the scope of this paper, but is going to be provided in an additional
} publication. 


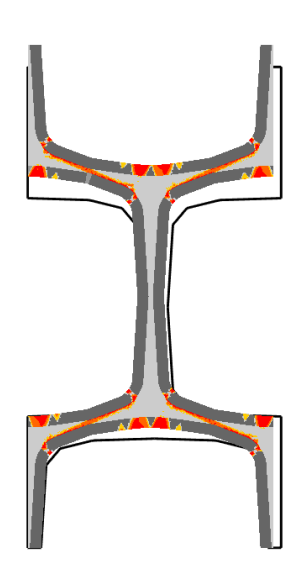

a

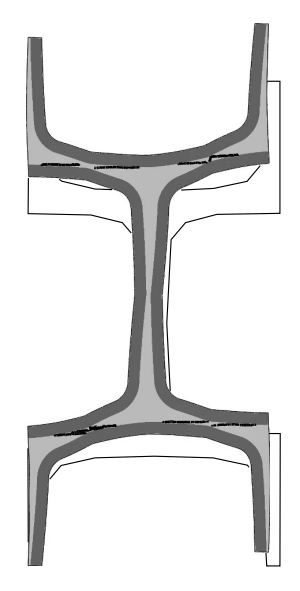

$\bar{a}$

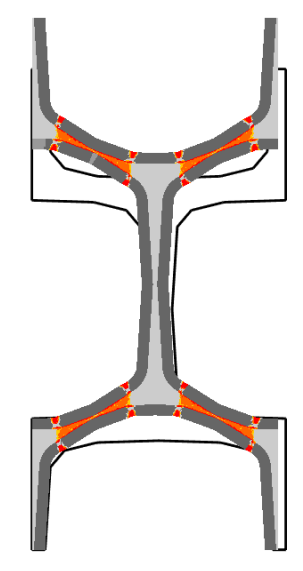

$b$

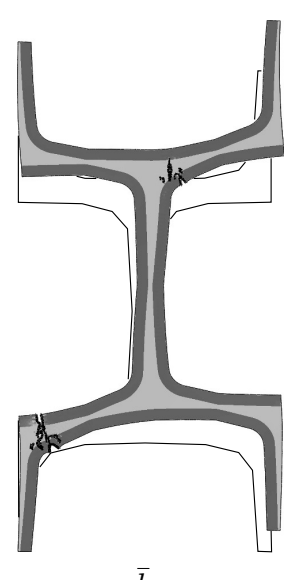

$\bar{b}$

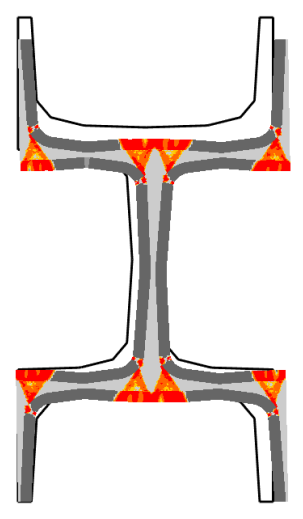

c

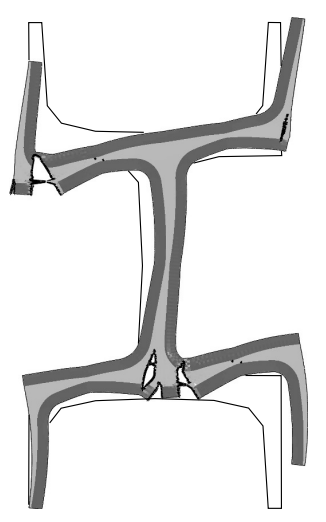

$\bar{c}$

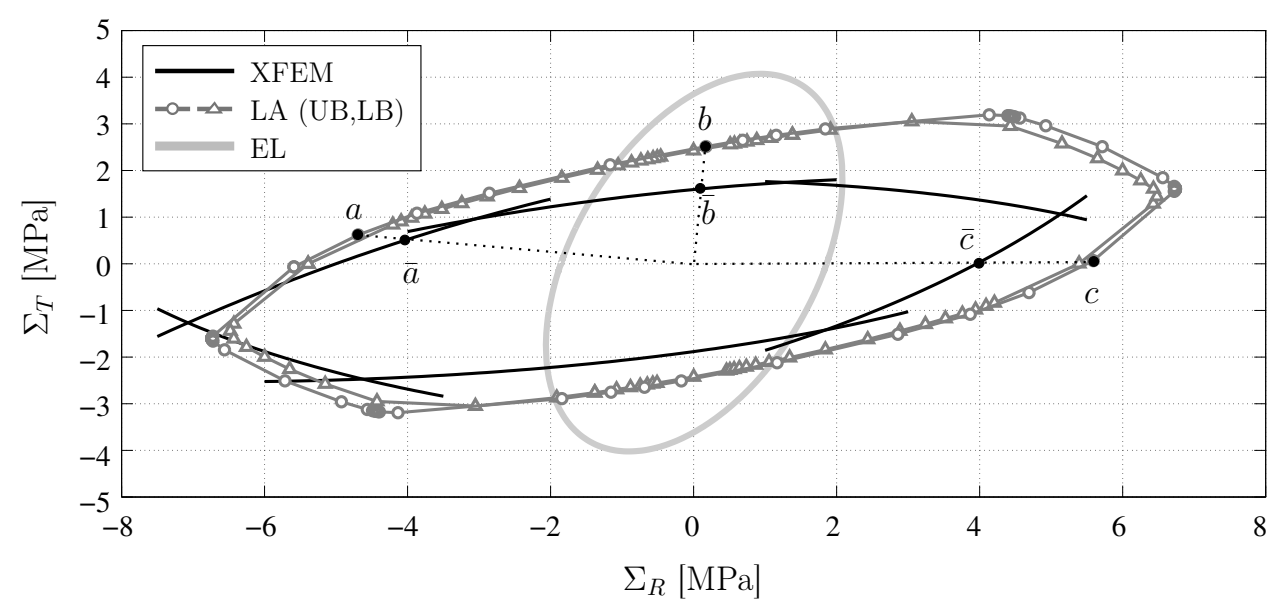

Figure 7: Effective failure surfaces for earlywood in the $R-T$ plane, obtained with the three different methods, and selected failure modes obtained with upper bound LA $(a, b$, and $c)$ and XFEM $(\bar{a}, \bar{b}$, and $\bar{c})$. 
ond effective strain indicating cellulosic fibre failure could be implemented, or higher order effective strain estimates could be envisaged.

The same procedure has been applied to the latewood unit cell, and the resulting effective failure surfaces are shown in Figure 8. Again, the obtained gaps between the LA lower and upper bounds are very small, giving reliable strength estimates for ductile failure. Due to the greater thickness of the latewood cell walls, a larger difference between ductile and brittle failure estimates could be expected, which is indeed reflected by comparing the effective failure surfaces obtained from LA and XFEM. Also, the related failure modes do not show such distinct correlations as for the earlywood cell. Nevertheless, the basic shape and orientation of the failure surfaces are still in good agreement. Since the overall volume of solid material in the latewood cell is significantly greater, high strain and stress concentrations in corner regions as well as the strengthening effect of cellulosic fibres play a minor role. For this reason, the effective failure surface obtained with the EL approach fits well into the overall result, predicting structural failure in between brittle and ductile failure, as its characteristics (cf. Figure 3) would suggest.

While the results at this observation level seem to be reliable, and give useful insights into the possibilities of the presented methods, they cannot be verified on the basis of experimental data. For this reason, the proposed methods have also been applied to the next higher observation scale, the clear wood scale, as described in Section 3.2. These results are presented in the following section.

\subsection{Clear wood scale}

To the knowledge of the authors, the only comprehensive experimental data set for multiaxial failure stress states of spruce wood can be found in [49], wherein different biaxial loading states in the $L$ - $R$ plane were applied to clear wood samples. To utilise these experimental results, the proposed computational methods have been used to obtain effective failure surfaces $F(\boldsymbol{\Sigma})$ for the same loading states as produced within biaxial testing. The rather complex test specimen and the load application mechanism are shown in Figure 9(a). Only clear wood without any growth irregularities and with linear and parallel annual rings was used for the specimens. The loading was applied under displacement control, and the biaxial strength of the sample was assumed to be reached as soon as a peak reaction force in one of the two directions could be identified. Both very brittle as well as ductile failure mechanisms were observed, but little hardening, so the experimental failure stress states plotted in Figure 10 (circular markers) approximately represent ultimate failure loads, regardless of their nature of failure.

The corresponding unit cell for the numerical approaches, obeying periodic boundary conditions, is schematically illustrated in Figure 9(b). When using the LA approach, this unit cell was discretised with about 700 triangular elements as described in Section 2.2, and around 800 different loading situations were applied to finally obtain the effective failure surfaces shown in Figure 10 (solid and dashed black lines) ${ }^{3}$. Again, the bound gap is satisfactorily small and a visually noticeable difference is only present for grain angles $\alpha$ between $7.5^{\circ}$ and $30^{\circ}$. Together with the small amount of computational effort, which was about 1 hour of computing time per failure surface on a commercial PC with four CPUs, the LA approach represents a remarkably efficient and stable tool for obtaining rigorous effective strength information (for ductile failure).

\footnotetext{
${ }^{3}$ Illustrative failure modes and a detailed presentation of all the LA results is beyond the scope of this paper, but is going to be provided in an additional publication.
} 


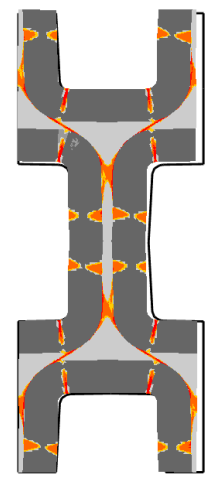

a

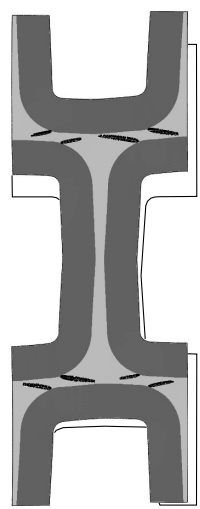

$\bar{a}$

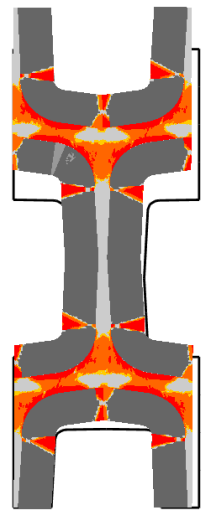

b

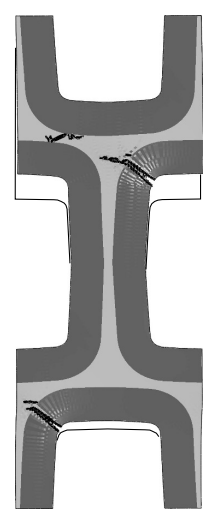

$\bar{b}$

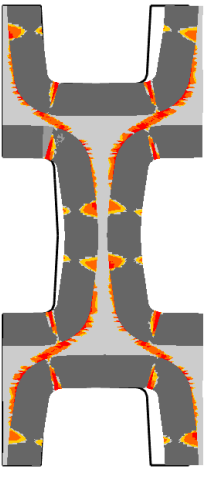

$c$

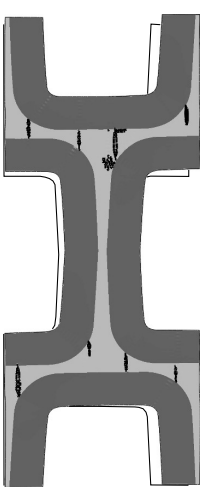

$\bar{c}$

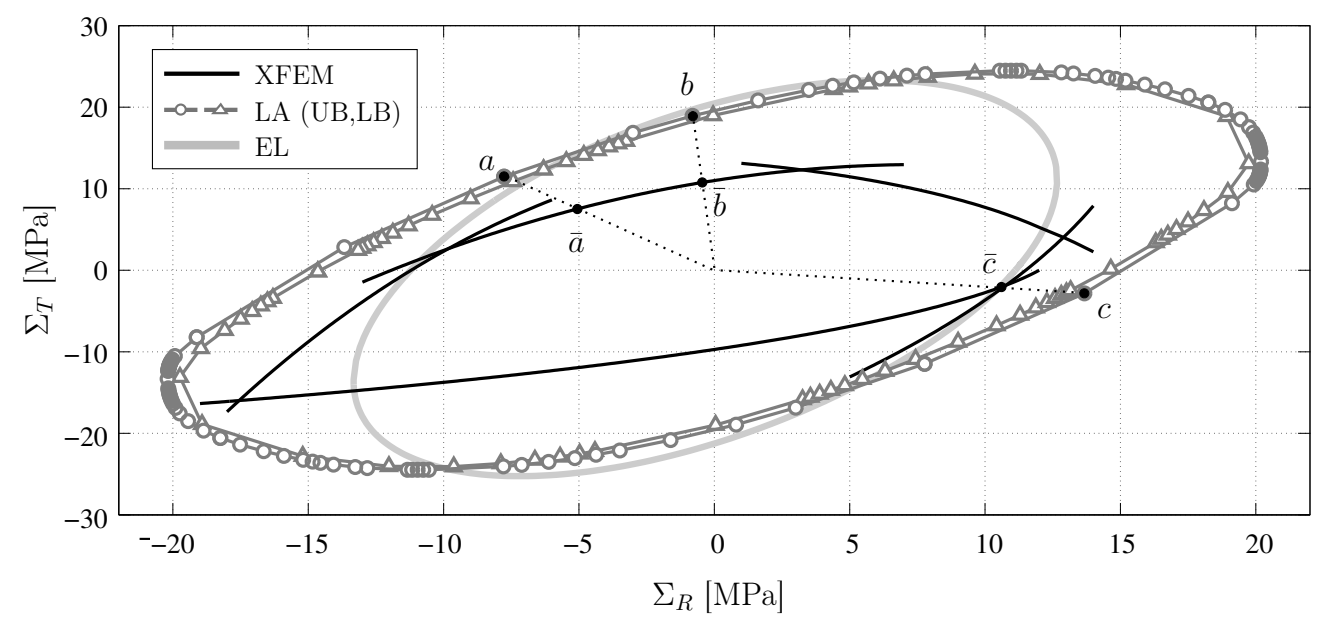

Figure 8: Effective failure surfaces for latewood in the $R-T$ plane, obtained with the three different methods, and selected failure modes obtained with upper bound LA $(a, b$, and $c)$ and XFEM $(\bar{a}, \bar{b}$, and $\bar{c})$. 


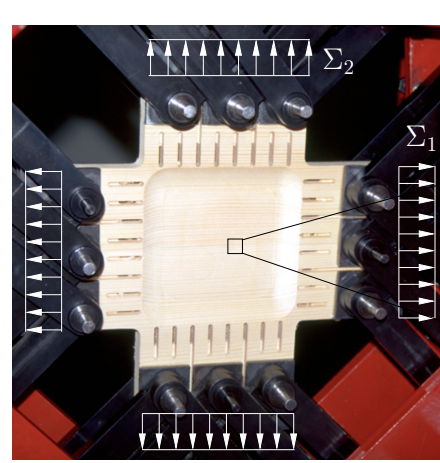

(a)

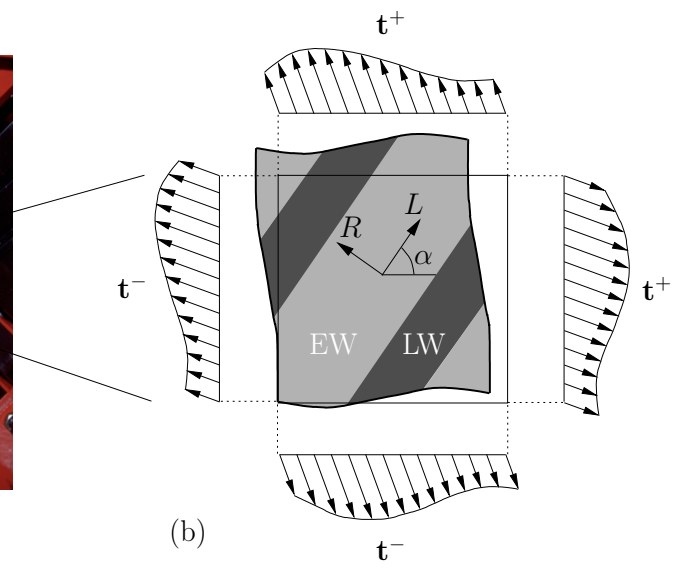

Figure 9: (a) Test specimen and load application mechanism for biaxial strength tests in [49], and (b) clear wood unit cell as used in the XFEM and LA approaches, showing layers of earlywood (EW) and latewood (LW).

In contrast, the XFEM calculations at this observation scale have proven to be rather difficult. The homogeneous structure of the unit cell (Figure 9(b)) and the resulting smooth stress and strain fields make it difficult to identify a distinct point for crack initiation. Additionally, the hard boundary between earlywood and latewood can cause numerical problems, but its proper consideration is essential to obtain reasonable results. The way in which these problems can be tackled, and how 3D multi-surface failure criteria can be achieved in a similar manner to that proposed in [1] for one observation scale below, will be presented in a subsequent work. However, the multisurface failure criteria for early and latewood has already been applied in [54] to simulate micro wedge splitting tests, where early and latewood were modelled as individual layers. A very good agreement with experimental results were obtained for both the estimation of the load peak and the post-peak behaviour. Additionally, the failure mechanisms of both cell types were represented very well, which is another confirmation/validation of the chosen approach/assumptions at this "mesoscale". The effective failure stress states obtained with XFEM shown in Figure 10 (bold crosses) represent selected results of this work for the considered $L-R$ space. Even if not enough results could be obtained to define clear effective failure surfaces, they allow for a qualitative comparison with the other computational methods and the experimental data.

A very satisfactory result is that all methods, without any empirical calibration, are able to predict the correct magnitude and orientation of the experimentally-obtained effective strength regions. The LA results almost perfectly form an outer envelope to these regions. Since no significant hardening effects were encountered during the experiments, linear-elastic perfectly-plastic failure should lead to the highest effective strength, which exactly corresponds to the underlying assumptions of LA. By means of the LA results, the interpretation of the experimental results can now be extended. As it was stated before, in Section 2: 'A structure does not need to exhibit perfect plasticity for the theoretical plastic collapse load based on the peak yield stress of each component to be approached closely. Rather, it is necessary that, at the point when a collapse mechanism forms under a particular loading, all those regions within the structure which are undergoing straining lie very close to the peak yield stress which they can achieve.' This statement 

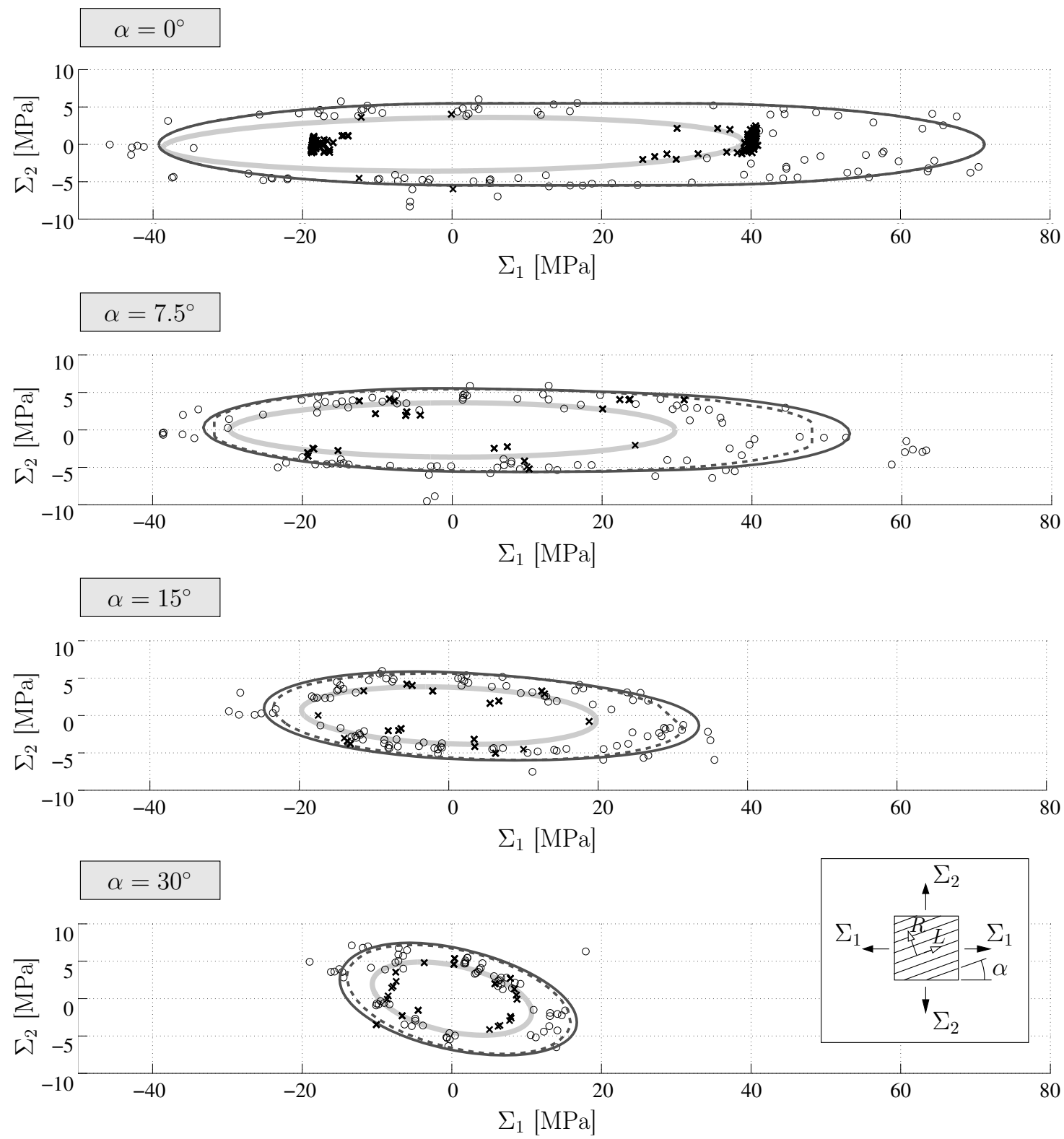

$\alpha=45^{\circ}$

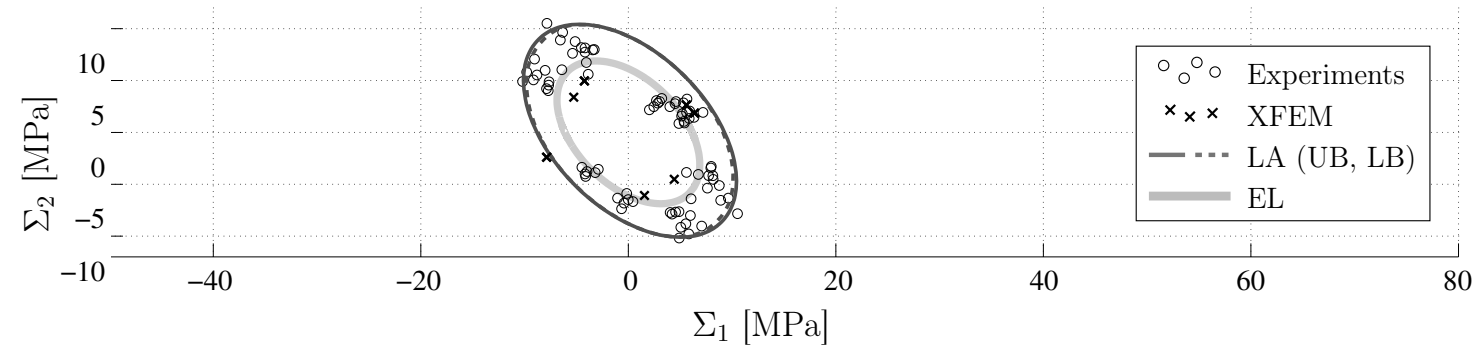

Figure 10: Failure stress states of clear wood in the $L-R$ plane for five different grain angles $\alpha$. Comparison of experimental results from [49] and effective failure surfaces obtained with the three different methods. 
may help explain the large fluctuation of the experimental strength values in tension-dominated regions. Even if the structural response of wood is known to be quite brittle under tensile loading, it seems that the microstructure of wood has a high capacity to redistribute stresses before ultimate failure. For this reason, the failure loads can (and do) reach values that would be expected for purely ductile failure. This supports the previously made assumption that wood has the ductile potential to 'activate' the strength of many points along a potential crack surface before brittle failure occurs. To what extent this potential can be exploited depends on how homogeneous and defect-free the considered specimen is. The natural variation of such defects may then introduce the experimentally-observed scatter of effective strengths.

The effective failure stress states obtained from XFEM are in good agreement with this argument, because they tend to reproduce the lowest strengths achieved in the experiments, and also deliver a larger scatter compared with the LA results. This corresponds well to the nature of brittle failure, where complex discrete cracks trigger the failure load and slightly different crack paths can lead to significantly different effective strength estimates.

Interestingly, the EL estimates deliver a very good inner envelope of the experimental results, and justify their name at this scale of observation. Since the underlying wood unit cell is chosen according to the mean mass density of the investigated clear wood, the strong underestimation of the earlywood strength in the $R$-direction (see Figure 7 ) is not observed at this scale. However, it is not accounted for that failure probably occurs mainly in the earlywood, the weaker phase at the clear wood scale, which would again lead to very low strength predictions. Nevertheless, even taking into account these weaknesses, the EL approach has the potential to be an ideally complementary method to the LA approach. And as Figure 10 demonstrates, together they could be highly effective tools for predicting the effective strength of wood under multidimensional stress states.

\section{Conclusions}

To assess the predictive capabilities of material models and computational approaches is almost impossible without an appropriate set of experimental data for validation. Even then, calibration parameters may shadow potential weaknesses, or inconsistencies may be traced back to the wrong origin. This has motivated the present work, in which three different computational methods have been applied to the same problem: the prediction of effective wooden strength behaviour. An extended finite element method (XFEM) approach able to reproduce brittle failure modes accurately, a newly-developed limit analysis (LA) approach capturing ductile failure modes, and an elastic limit (EL) approach based on continuum micromechanics, have all been applied to representative earlywood and latewood unit cells as well as clear wood structures. At both scales of observation (annual ring scale and clear wood scale) the geometric models and input parameters to all approaches have been chosen in a consistent way, to make sure that differences appearing in the results can mainly be assigned to the methods themselves.

Based on the results obtained, the three computational methods can be generally evaluated as follows:

- The XFEM approach is by far the most extensive with respect to computational effort and algorithmic implementation. This is not surprising, since this method includes the largest description flexibility, and thus has the potential to reproduce the processes in nature most realistically. It will not, however, 
be available for quick engineering estimates or for the build-up of material databases for wood species or products in the near future. Nevertheless, as a tool to gain knowledge of the mechanical behaviour of wood, and to serve as a reference for other wood-related models, it is of very great value.

- The LA approach has been successfully applied to wooden unit cell structures at both annual ring scale and clear wood scale. The necessary periodic boundary conditions, as well as anisotropic strength behaviour of certain material phases, were taken into account appropriately. Compared with XFEM, basic characteristics of failure modes and the overall strength behaviour could be captured correctly, both qualitatively and quantitatively. Along with the observed computational efficiency and numerical stability, these results render the LA approach as a very promising tool for this kind of application in the future.

- The continuum micromechanical basis of the EL approach makes it unbeatable with respect to efficiency and the elegance with which material properties of different phases can be linked across several scales of observation. However, when it comes to strength estimates, stress and strain averages over material phases (even if they are consistent with energy considerations) are perhaps not able to capture all failure-triggering processes. In particular, morphological aspects causing high stress or strain gradients may lead to prediction inaccuracies. Nevertheless, the potential of this method is undisputed and due to its efficiency it may become invaluable for rapid strength predictions within engineering design processes.

With respect to wood, a combination of the LA and EL approaches could evolve to an effective bundle of complementary methods, delivering 'inner' and 'outer' predictions for the natural scatter of wooden strengths. In wood products where brittle failure is prevented by reinforcements, and thus, the ductile potential of wood can be substantially activated, LA approaches may even be able to deliver reliable strength estimates on their own.

Another important motivation of this work has been to get insights into the potential of multisurface concepts which are able to provide detailed information about the 3D strength behaviour of clear wood. Information which cannot be achieved with identification experiments only, but which is required for the definition of realistic orthotropic failure envelopes at the clear wood scale, independent on their mathematical description. By relating strength behaviour to its microstructural origin, where failure processes are much more 'universal' with respect to different wood species, the number of strength properties to be identified for an accurate description of wood should be greatly reduced. Finally, reliable 2D and 3D strength information for wooden boards as well as wood-based products could be obtained in an efficient way, which would subsequently help to exploit their full potential in modern engineering structures.

Future work will be devoted to the extension of the LA approach to 3D, and its application to wooden boards and wood-based engineering products. Particularly important topics in this respect are layered wooden structures, as discussed in [55], and cross-laminated timber plates [56]. Preliminary 3D results agree very well with the $2 \mathrm{D}$ results presented within this work, and thus strengthen the statements made. Moreover, stochastic aspects as described in [57, 58], very relevant in layered wood-based products, will be taken into account in future works. 


\section{Acknowledgments}

We gratefully acknowledge the financial support of this work by the Austrian Science Fund (FWF) through the Erwin Schrödinger Fellowship J3748-N30.

\section{References}

[1] Lukacevic M, Füssl J, Lampert R (2015) Failure mechanisms of clear wood identified at wood cell level by an approach based on the extended finite element method. Engineering Fracture Mechanics 144:158-175. 10.1016/j.engfracmech.2015.06.066

[2] Lukacevic M, Füssl J. (2016) Application of a multisurface discrete crack model for clear wood taking into account the inherent microstructural characteristics of wood cells. Holzforschung. 10.1515/hf-2015-0162

[3] Hofstetter K, Hellmich Ch, Eberhardsteiner J, Mang HA (2008) Micromechanical estimates for elastic limit states in wood, revealing nanostructural failure mechanisms. Mechanics of Advanced Materials and Structures 15:474-484.

[4] Francescato P, Pastor J (1997) Limit analysis and homogenization: Predicting limit loads of periodic heterogeneous materials. European Journal of Mechanics - A/Solids 16, 235253.

[5] Füssl J, Lackner R, Eberhardsteiner J, Mang HA (2008) Failure modes and effective strength of two-phase materials determined by means of numerical limit analysis. Acta Mechanica 1(195):185-202. 10.1007/s00707-007-0550-9

[6] Pastor J, Thore Ph, Pastor F (2010) Limit analysis and numerical modeling of spherically porous solids with Coulomb and Drucker-Prager matrices. Journal of Computational and Applied Mathematics 234, 21622174.

[7] Masuda M (1988) Theoretical consideration on fracture criteria of wood - proposal of finite small area theory. Proceedings of the 1988 International Conference on Timber Engineering, Seattle, Vol 2:584-595.

[8] Landelius J (1989) Finit area metoden. en bra metod för beräkning av uppfläkningsbrott? Rep No TVSM 5043:66, in Swedish.

[9] Aicher S, Gustafsson PJ, Haller P, Petersson H (2002) Fracture mechanics models for strength analysis of timber beams with a hole or a notch - a report of rilem tc-133.

[10] Serrano E, Gustafsson J (2006) Fracture mechanics in timber engineering - strength analyses of components and joints. Material and Structures 40:87-96.

[11] Sjödin J, Serrano E (2008) A numerical study of methods to predict the capacity of multiple steel-timber dowel joints. Holz als Roh- und Werkstoff 66(6):447-454.

[12] Sjödin J, Serrano E, Enquist B (2008) An experimental and numerical study of the effect of friction in single dowel joints. Holz als Roh- und Werkstoff 66(5):363-372.

[13] Guindos P (2011) Three-dimensional finite element models to simulate the behavior of wood with presence of knots, applying the flow-grain analogy and validation with close range photogrammetry. PhD thesis, University of Santiago de Compostela, Department of Agroforestry Engineering.

[14] Lukacevic M, Füssl J (2014) Numerical simulation tool for wooden boards with a physically based approach to identify structural failure. European Journal of Wood and Wood Products 72(4):497-508. 10.1007/s00107-014-0803-y

[15] Lukacevic M, Füssl J, Griessner M, Eberhardsteiner J (2014) Performance assessment of a numerical simulation tool for wooden boards with knots by means of full-field deformation measurements. Strain 50(4):301-317. 10.1111/str.12093

[16] Mackenzie-Helnwein P, Eberhardsteiner J, Mang HA (2003) A multi-surface plasticity model for clear wood and its application to the finite element analysis of structural details. Computational Mechanics 31:204-218.

[17] Schmidt J, Kaliske M (2007) Simulation of cracks in wood using a coupled material model for interface elements. Holzforschung $61(4): 382-389$.

[18] Schmidt J, Kaliske M (2009) Models for numerical failure analysis of wooden structures. Engineering Structures 31(2):571579.

[19] Danielsson H, Gustafsson PJ (2014) Fracture analysis of glued laminated timber beams with a hole using a 3D cohesive zone model. Engineering Fracture Mechanics 124-125:182-195.

[20] Denton SR, Morley CT (2000) Limit analysis and strain-softening structures. International Journal of Mechanical Sciences 42:503-522.

[21] Zaoui A (2002) Continuum micromechanics: Survey. ASCE Journal of Engineering Mechanics 128(8):808-816.

[22] Suquet P (1997) Continuum Micromechanics. Springer Verlag, Wien, New York. 
[23] Belytschko T, Black T (1999) Elastic crack growth in finite elements with minimal remeshing. International Journal for Numerical Methods in Engineering 45(5):601-620.

[24] Melenk J, Babuska I (1996) The partition of unity finite element method: Basic theory and applications. Computer Methods in Applied Mechanics and Engineering 139(1):289-314.

[25] Osher S, Sethian JA (1988) Fronts propagating with curvature-dependent speed: Algorithms based on hamilton-jacobi formulations. Journal of Computational Physics 79(1):12-49.

[26] Moës N, Gravouil A, Belytschko T (2002) Non-planar 3d crack growth by the extended finite element and level sets-part i: Mechanical model. International Journal for Numerical Methods in Engineering 53(11):2549-2568.

[27] Gravouil A, Moës N, Belytschko T (2002) Non-planar 3d crack growth by the extended finite element and level sets-part ii: Level set update. International Journal for Numerical Methods in Engineering 53(11):2569-2586.

[28] Song JH, Areias PMA, Belytschko T (2006) A method for dynamic crack and shear band propagation with phantom nodes. International Journal for Numerical Methods in Engineering 67(6):868-893.

[29] Hansbo A, Hansbo P (2004) A finite element method for the simulation of strong and weak discontinuities in solid mechanics. Computer Methods in Applied Mechanics and Engineering 193(33-35):3523-3540.

[30] Drucker DC, Greenberg HJ, Prager W (1951) The safety factor of an elastic-plastic body in plane strain. Journal of Applied Mechanics 18:371-378.

[31] Drucker DC, Prager W, Greenberg HJ (1952) Extended limit design theorems for continuous media. Quarterly of Applied Mathematics 9:381-389.

[32] Lubliner J (1990) Plasticity Theory. University of California Berkeley. Revised Pdf Edition 2006. Previously published by Pearson Eduction, Inc.

[33] Makrodimopoulos A, Martin CM (2005) Limit analysis using large-scale socp optimization. Proc. 13th Nat. Conf. of UK Association for Computational Mechanics in Engineering, Sheffield, Pages 21-24.

[34] Makrodimopoulos A (2010) Remarks on some properties of conic yield restrictions in limit analysis. International Journal for Numerical Methods in Biomedical Engineering 26(11):1449-1461.

[35] Makrodimopoulos A, Martin CM (2005) A novel formulation of upper bound limit analysis as a second-order cone programming problem. Proc. 8th Int. Conf. on Computational Plasticity, Barcelona, Pages 1083-1086.

[36] Makrodimopoulos A, Martin CM (2007) Upper bound limit analysis using simplex strain elements and second-order cone programming. International Journal for Numerical and Analytical Methods in Geomechanics 31(6):835-865.

[37] Andersen ED, Roos C, Terlaky T (2003) On implementing a primal-dual interior-point method for conic quadratic optimization. Mathematical Programming, Series B 95:249-277.

[38] MOSEK ApS (2006) The MOSEK optimization tools version 4.0 (revision 35). User's Manual and Reference, available from http://www.mosek.com.

[39] Suquet P (1987) Elements of homogenization for inelastic solid mechanics. Homogenization Techniques for Composite Media. Eds. Sanchez-Palencia E, Zaoui A, Lecture Notes in Physics 272. Springer Verlag, Wien/New York. Pages 193278.

[40] Benveniste Y (1987) A new approach to the application of Mori-Tanaka's theory in composite materials. Mechanics of Materials 6:147-157.

[41] Eshelby JD (1957) The determination of the elastic field of an ellipsoidal inclusion, and related problems, Proc. R. Soc. London Ser. A 241:376-396.

[42] Laws N (1977) The determination of stress and strain concentrations at an ellipsoidal inclusion in an anisotropic material, Journal of Elasticity 7(1):91-97.

[43] Böhm H (2004) A short introduction to continuum micro-mechanics. Mechanics of Microstructured Materials. Ed. Böhm H. CISM Lecture Notes 464. Springer Verlag, Wien/New York. Pages 1-40.

[44] Hofstetter K, Hellmich Ch, Eberhardsteiner J (2006) Micromechanical modeling of solid-type and plate-type deformation patterns within softwood materials. A review and an improved approach. Holzforschung 61:343-351.

[45] Hill R (1951) On the state of stress in a plastic-rigid body at the yield point. Philosophical Magazine 42:868-875.

[46] Eberhardsteiner J, Hofstetter K, Hellmich Ch (2005) Development and experimental validation of a continuum micromechanics model for the elasticity of wood. European Journal of Mechanics - A/Solids 24:1030-1053.

[47] Bader T, Hofstetter K, Hellmich C, Eberhardsteiner J (2011) The poroelastic role of water in cell walls of the hierarchical composite softwood. Acta Mechanica 217:75-100. 
[48] Bader T, Hofstetter K, Hellmich C, Eberhardsteiner J (2010) Poromechanical scale transitions of failure stresses in wood: From the lignin to the spruce level. ZAMM - Zeitschrift für angewandte Mathematik und Mechanik 90:750-767.

[49] Eberhardsteiner J (2002) Mechanisches Verhalten von Fichtenholz: Experimentelle Bestimmung der biaxialen Festigkeitseigenschaften, Mechanical Behavior of Spruce Wood: Experimental Determination of Biaxial Strength (Properties), in German (Springer Verlag, Wien, New York).

[50] Zimmermann T, Sell J, Eckstein D (1994) Rasterelektronenmikroskopische Untersuchungen an Zugbruchflächen von Fichtenholz. Holz als Roh- und Werkstoff 52(4):223-229.

[51] Schwiedrzik J, Raghaven R, Rüggeberg M, Hansen S, Wehrs J, Adusumalli RB, Zimmermann T, Michler J (2016) Identification of polymer matrix yield stress in the wood cell wall based on micropillar compression and micromechanical modelling. Philosophical Magazine 96:32-34, 3461-3478.

[52] Puck A, Schuermann H (1998) Failure analysis of frp laminates by means of physically based phenomenological models. Composites Science and Technology 58(7):1045-1067.

[53] Tsai SW, Wu EM (1971) A general theory of strength for anisotropic materials. Journal of Composite Materials 5(1):5880.

[54] Lukacevic M, Füssl J (2016) Application of a multisurface discrete crack model for clear wood taking into account the inherent microstructural characteristics of wood cells. Holzforschung 70(9):845-853.

[55] Gaff M, Gasparik M, Boruvka V, Haviarova E (2015) Stress simulation in layered wood-based materials under mechanical loading. Materials and Design 87:10651071.

[56] Hochreiner G, Füssl J, Eberhardsteiner J (2014) Cross-Laminated Timber Plates Subjected to Concentrated Loading Experimental Identification of Failure Mechanisms. Strain 50:68-81.

[57] Kandler G, Füssl J, Eberhardsteiner J (2015) Stochastic Finite Element Approaches for Wood-Based Products: Theoretical Framework and Review of Methods. Wood Science and Technology 49:1055 - 1097.

[58] Füssl J, Kandler G, Eberhardsteiner J (2016) Application of Stochastic Finite Element Approaches to Wood-Based Products. Archive of Applied Mechanics, 86(1-2):89-110. 